\title{
Reelin Is Required for Class-Specific Retinogeniculate Targeting
}

\author{
Jianmin Su, ${ }^{1}$ Cheryl V. Haner, ${ }^{1}$ Terence E. Imbery, ${ }^{1}$ Justin M. Brooks, ${ }^{1}$ Duncan R. Morhardt, ${ }^{2}$ Karen Gorse, ${ }^{1}$ \\ William Guido, ${ }^{1}$ and Michael A. Fox ${ }^{1}$ \\ Departments of ${ }^{1}$ Anatomy and Neurobiology and ${ }^{2}$ Biochemistry and Molecular Biology, Virginia Commonwealth University Medical Center, Richmond, \\ Virginia 23298-0709
}

Development of visual system circuitry requires the formation of precise synaptic connections between neurons in the retina and brain. For example, axons from retinal ganglion cells (RGCs) form synapses onto neurons within subnuclei of the lateral geniculate nucleus (LGN) [i.e., the dorsal LGN (dLGN), ventral LGN (vLGN), and intergeniculate leaflet (IGL)]. Distinct classes of RGCs project to these subnuclei: the dLGN is innervated by image-forming RGCs, whereas the vLGN and IGL are innervated by non-image-forming RGCs. To explore potential mechanisms regulating class-specific LGN targeting, we sought to identify differentially expressed targeting molecules in these LGN subnuclei. One candidate targeting molecule enriched in the vLGN and IGL during retinogeniculate circuit formation was the extracellular matrix molecule reelin. Anterograde labeling of RGC axons in mutant mice lacking functional reelin $\left(\right.$ reln $\left.^{\mathrm{r} l / \mathrm{rl}}\right)$ revealed reduced patterns of vLGN and IGL innervation and misrouted RGC axons in adjacent non-retino-recipient thalamic nuclei. Using genetic reporter mice, we further demonstrated that mistargeted axons were from non-image-forming, intrinsically photosensitive RGCs (ipRGCs). In contrast to mistargeted ipRGC axons, axons arising from image-forming RGCs and layer VI cortical neurons correctly targeted the dLGN in reln $^{\mathrm{r} / \mathrm{rl} \mathrm{l}}$ mutants. Together, these data reveal that reelin is essential for the targeting of LGN subnuclei by functionally distinct classes of RGCs.

\section{Introduction}

Retinal ganglion cells (RGCs), the output neurons of the retina, project axons to over 20 distinct CNS nuclei. Because of the complexity of visual information conveyed by retinal inputs (e.g., color, contrast, light intensity, and object movement), RGC axons are targeted to domains within retino-recipient nuclei by at least three distinct mechanisms. The best characterized of these targeting mechanisms involves the sorting of RGC axon terminals into topographically arranged maps within a retino-recipient nucleus. Such maps convey the position of information in the visual field to a spatially correlated region of a retino-recipient nucleus. Wnts, BMPs (bone morphogenetic proteins), ephrins/Ephs, ALCAM (activated leukocyte cell adhesion molecule), and L1 each have well documented roles in the targeting RGC axons into these retinotopic maps in mouse (Hindges et al., 2002; Demyanenko and Maness, 2003; Pfeiffenberger et al., 2005; Sakuta et al., 2006; Schmitt et al., 2006; Buhusi et al., 2009) (for review, see Huberman et al., 2008a; Sanes and Yamagata, 2009).

\footnotetext{
Received Aug. 11, 2010; revised 0ct. 7, 2010; accepted Nov. 1, 2010.

This work was supported by The Thomas F. Jeffress and Kate Miller Jeffress Memorial Trust (M.A.F.) and National Institutes of Health (NIH)-National Eye Institute Grant EY012716 (W.G.). Microscopy was performed at the Virginia Commonwealth University Department of Anatomy and Neurobiology Microscopy Facility supported, in part, with funding from NIH-National Institute of Neurological Disorders and Stroke Center Core Grant 5P30 NS047463-02. We thank Dr. J. R. Sanes for comments on this manuscript and for providing anti- $\beta$-galactosidase antibodies. We thank Drs. S. Hattar and A. T. Campagnoni for providing opn4-tau-LaCZ and golli-tau-GFP mice, respectively, and Dr. C. K. Chen for providing anti-melanopsin antibodies.

Correspondence should be addressed to Michael A. Fox, 1101 East Marshall Street, P.0. Box 98-0709, Richmond, VA23298-0709.E-mail:mafox@vcu.edu.

DOI:10.1523/JNEUROSCI.4227-10.2011

Copyright $\odot 2011$ the authors $\quad 0270-6474 / 11 / 310575-12 \$ 15.00 / 0$
}

In addition to being retinotopically mapped, retinal inputs are sorted into eye-specific domains within retino-recipient nuclei (Muscat et al., 2003; Huberman et al., 2008a; Sanes and Yamagata, 2009; Sanes and Zipursky, 2010). In mouse lateral geniculate nucleus (LGN), ephrins/Ephs and teneurins contribute to the initial patterning of eye-specific retinal input (Pfeiffenberger et al., 2005; Leamey et al., 2007; Rebsam et al., 2009). Subsequently, MHC1, C1q, and neuronal pentraxins-in combination with neuronal activity - contribute to the refinement of these inputs into non-overlapping eye-specific domains (Huh et al., 2000; Bjartmar et al., 2006; Stevens et al., 2007; Datwani et al., 2009; Feller, 2009).

Last, RGCs are grouped into functional and morphological classes and axons from each class target specific retino-recipient nuclei or specific domains within retino-recipient nuclei (Masland, 2001; Völgyi et al., 2009; Sanes and Zipursky, 2010). Although projection patterns of several classes of mouse RGCs have recently been reported (Hattar et al., 2006; Huberman et al., 2008b, 2009; Kim et al., 2008, 2010), the mechanisms regulating class-specific targeting remain unclear. To address this issue, we sought to identify molecular cues responsible for class-specific targeting of RGC axons in mouse LGN-a retino-recipient nucleus where class-specific targeting is most evident. The LGN is comprised of three functionally distinct subnuclei-the dorsal LGN (dLGN), the ventral LGN (vLGN), and the intergeniculate leaflet (IGL). Whereas the dLGN receives image-processing retinal input (Hale and Sefton, 1978; Lüth et al., 1993; Huberman et al., 2008b, 2009; Kim et al., 2008), the vLGN and IGL receive non-image-forming input from intrinsically photosensitive 
RGCs (ipRGCs) (Hattar et al., 2002, 2006; Morin et al., 2003) (see Fig. $1 A, B)$. By screening for differentially expressed targeting cues within these LGN subnuclei, we identified several candidates that may contribute to class-specific retinogeniculate targeting. Here, we provide compelling evidence that one candidate-the extracellular matrix (ECM) molecule reelin—is essential for LGN subnuclei-specific targeting by ipRGC axons, but not by axons originating from image-forming RGCs.

\section{Materials and Methods}

Antibodies. A rabbit polyclonal antibody direct against melanopsin (Meln) was kindly provided by Dr. C. K. Chen (Virginia Commonwealth University, Richmond, VA) (diluted 1:1000 for whole mounts and 1:2500 for cryosections). A rabbit polyclonal antibody directed against $\beta$-galactosidase (LacZ) was kindly provided by Dr. J. R. Sanes (Harvard University, Cambridge, MA) (diluted 1:500). Antibodies for the following antigens were purchased: rabbit anti-calretinin (Millipore; diluted 1:1000), rabbit anti-vesicular glutamate transporter 2 (VGLUT2) (Synaptic Systems; diluted 1:500), rabbit anti-green fluorescent protein (GFP) (Invitrogen; diluted 1:250), mouse anti-reelin (Abcam; diluted 1:1000), rabbit anti-neuropeptide Y (NPY) (ImmunoStar; diluted 1:500), mouse anti-neuronal nuclei (NeuN) (Millipore; 1:200), rabbit anti-glutamate decarboxylase 65/67 (Gad65/67) (Millipore Bioscience Research Reagents; 1:500), mouse anti-SMI32 (Covance; 1:500), and rabbit anti-disabled-1 (Dab1) (Abnova; diluted 1:500). Fluorescently conjugated secondary antibodies were purchased from Invitrogen or Jackson ImmunoResearch (diluted 1:1000).

Mice. Wild-type C57 and CD1 mice were from Charles River. Reeler mutant mice $\left(r e l n^{\mathrm{rl} / \mathrm{rl}}\right)$ and scrambler mutants $\left(\mathrm{dab} 1^{\mathrm{scm} / \mathrm{scm}}\right)$ were obtained from The Jackson Laboratory. The generation of opn4-tau-LacZ and golli-tau-GFP mice were described previously (Hattar et al., 2002; Jacobs et al., 2007). Genomic DNA was isolated and genotyping performed as previously described (Su et al., 2010). The following primer pairs were used: reln, TTA ATC TGT CCT CAC TCT GCC CTC T and GCA GAC TCT CTT ATT GTC TCT AC; mutant reln, TTA ATC TGT CCT CAC TCT GCC CTC T and TTC CTC TCT TGC ATC CTG TTT TG (Yamamoto et al., 2003); lacZ, TTC ACT GGC CGT CGT TTT ACA ACG TCG TGA and ATG TGA GCG AGT AAC AAC CCG TCG GAT TCT; $g f p$, AAG TTC ATC TGC ACC ACC G and TCC TTG AAG AAG ATG GTG CG. Scrambler mutants were identified by their ataxic gait or their malformed CNS. All analyses conformed to National Institutes of Health guidelines and protocols approved by the Virginia Commonwealth University Institutional Animal Care and Use Committee.

Immunohistochemistry. Immunohistochemistry (IHC) was performed on $16 \mu \mathrm{m}$ coronal cryosectioned tissues as described previously ( $\mathrm{Su}$ et al., 2010 ) or on whole-mount retinal preparations. For whole-mount staining, retinas were dissected and postfixed in $4 \%$ paraformaldehyde in PBS. Retinas were washed, incubated in blocking buffer (2.5\% BSA, 5\% NGS, $0.1 \%$ Triton X-100 in PBS), and subsequently incubated with antibodies diluted in blocking buffer for $24-48 \mathrm{~h}$ at $4^{\circ} \mathrm{C}$. After washing in PBS and incubation with secondary antibodies (diluted in blocking buffer), retinas were mounted on SuperFrost Plus slides (Thermo Fisher Scientific) with VectaSheild (Vector Laboratories). Colabeling with antibodies from the same host species was performed with a Zenon labeling kit (Invitrogen). Images were acquired on a Zeiss AxioImager A1 fluorescent microscope or a Leica SP2 confocal microscope. When comparing different ages of tissues or between genotypes, images were acquired with identical parameters. For quantifying numbers of ipRGCs in retinal whole mounts, $5-10$ random images $(875 \times 650 \mu \mathrm{m})$ were acquired on a Zeiss AxioImager A1 from control or reln ${ }^{\mathrm{r} l / \mathrm{rl}}$ mutant retinas. All melanopsinexpressing ipRGCs in these images were counted manually. A total of 11 control retinas and 12 mutants retinas were analyzed. For quantifying the spatial extent of M1 ipRGC arborization into mutant $\left(\right.$ reln $^{\mathrm{r} / \mathrm{rl}}$ and dab $\left.1^{\mathrm{scm} / \mathrm{scm}}\right)$ dLGN, images of LacZ immunoreactivity were acquired from postnatal day 21 (P21) opn4-tau-Lac ${ }^{+/+}$mice, reln ${ }^{\text {rl/rl }} ;$ opn4-tau$\mathrm{LacZ}^{+/+}$mutants, and dab1 $1^{\mathrm{scm} / \mathrm{scm}} ;$ opn $4-$ tau-LacZ ${ }^{+/-}$mice. The total area of dLGN and the area of dLGN containing LacZ immunoreactivity were measured with AxioVision software (Carl Zeiss Imaging Systems).
A minimum of three animals (per genotype and per age) were compared in all IHC experiments.

In situ hybridization. In situ hybridization (ISH) was performed on $10-16 \mu \mathrm{m}$ coronal cryosectioned tissues as described previously ( $\mathrm{Su}$ et al., 2010). The generation of syt1 and syt2 riboprobes was previously described (Fox and Sanes, 2007). Using a similar protocol, riboprobes were generated from adamts15, vldlr, and apoer2 IMAGE clones (clone IDs 30619053, 3968213, 40109899, respectively) (Open Biosystems). A minimum of three animals per genotype and age was compared in ISH experiments.

Microarray analysis. LGN subnuclei were isolated from P3 vLGN and IGL (vLGN/IGL) or dLGN. Mice were decapitated, brains were removed, and $300 \mu \mathrm{m}$ coronal sections were cut in ice-cold DEPC-PBS with a vibratome. vLGN/IGL or dLGN were microdissected and tissues from at least five littermates were pooled per sample. RNA was isolated using the Bio-Rad Total RNA Extraction from Fibrous and Fatty Tissue kit (BioRad). RNA purity assessment, first- and second-strand cDNAs preparation, cRNAs generation, hybridization to Agilent Whole Genome $44 \mathrm{kx} 4$ mouse arrays, and data analysis with Agilent Feature Extraction and GeneSpring GX, version 7.3.1, software packages were performed by GenUs Biosystems. To be considered differentially expressed genes must have been twofold higher in the averaged sample sets $(n=3 ; p<0.05)$. Three samples were analyzed per region.

Quantitative PCR. RNA was purified from pooled samples isolated from P3, P6, P8, P10, and P14 vLGN/IGL or dLGN as described above. cDNAs were generated with Superscript II Reverse Transcriptase FirstStrand cDNA Synthesis kit (Invitrogen). Quantitative PCR (qPCR) was performed on a Chromo4 Four-Color Real-Time System (Bio-Rad) using iQ SYBR Green Supermix (Bio-Rad) as described previously ( $\mathrm{Su}$ et al., 2010). The following primer pairs were used: actin, TTC TTT GCA GCT CCT TCG TT and ATG GAG GGG AAT ACA GCC C; reln, CTT CTC AGA GCA TTG GAG GC and ACA TGA GAG GCC ACC ACA CT; slit2, TTC AGT TGT TTC CTG AGC TGC and CCT TCC TTG GAA TTG CTT GA; thbs4, AAT TCA CTG TGA TGG GAC GG and CAG CCA GCT GCA AGT TGT T; sema3c, TGT ACG AGG ATC TTC CCA GC and CTG CTG GTG GGA CAG ACT AA. A minimum of four experiments (each in triplicate) was run for each gene, at each age examined. Each individual run on the Chromo4 Four-Color Real-Time System included separate actin controls.

Intraocular injections of anterograde tracers. Intraocular injection of cholera toxin subunit B (CTB) conjugated to Alexa Fluor 488 or Alexa Fluor 594 (Invitrogen) was performed as described previously (JaubertMiazza et al., 2005). After 1-2 d, mice were killed, and brains were fixed in $4 \%$ paraformaldehyde. Eighty to $100 \mu \mathrm{m}$ coronal sections were sectioned on a vibratome and mounted in ProLong Gold (Invitrogen). Retinal projections were analyzed from at least five animals for each age and genotype. Images were acquired on a Leica SP2 confocal microscope. To quantify the spatial extent of vLGN and IGL innervation by retinal axons, serial coronal sections encompassing the entire LGN $(\sim 14-1880 \mu \mathrm{m}$ sections) were obtained and imaged from six P12 $\mathrm{reln}^{\mathrm{rl} / \mathrm{rl}}$ mutants and six littermate controls (for example, see serial sections shown in supplemental Fig. S4, available at www.jneurosci.org as supplemental material). Measurements of the entire LGN area and the area of retinal innervation to vLGN and IGL in mutants and controls were obtained using AxioVision software.

Pupillary light reflexes. After $1 \mathrm{~h}$ of dark adaptation, mice $(n=3$ per genotype) were restrained and one eye monitored under infrared light with a Sony DCR-HC96 camera. Pupillary light reflexes (PLRs) were evoked by $30 \mathrm{~s}$ of high-intensity light $\left(1.7 \mathrm{~mW} / \mathrm{cm}^{2}\right)$ from a $473 \mathrm{~nm}$ light-emitting diode. Video frames were captured for $20 \mathrm{~s}$ before the application of light and during the $30 \mathrm{~s}$ burst of low-intensity light. Pupil size was measured from video images before the onset of light and at the end of the $30 \mathrm{~s}$ burst of light.

\section{Results}

Identification of nuclei-specific candidate targeting cues

To address how functionally distinct classes of RGC axons target distinct domains within retino-recipient nuclei, we sought to identify the mechanisms regulating class-specific targeting of the 
A

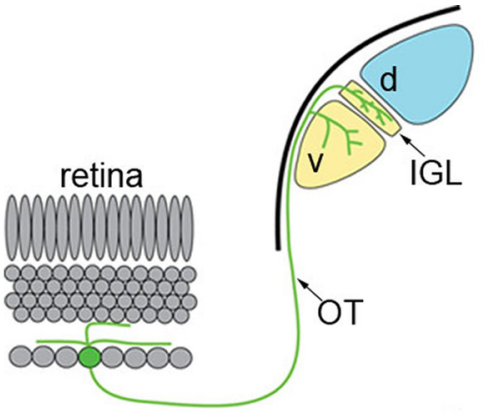

C

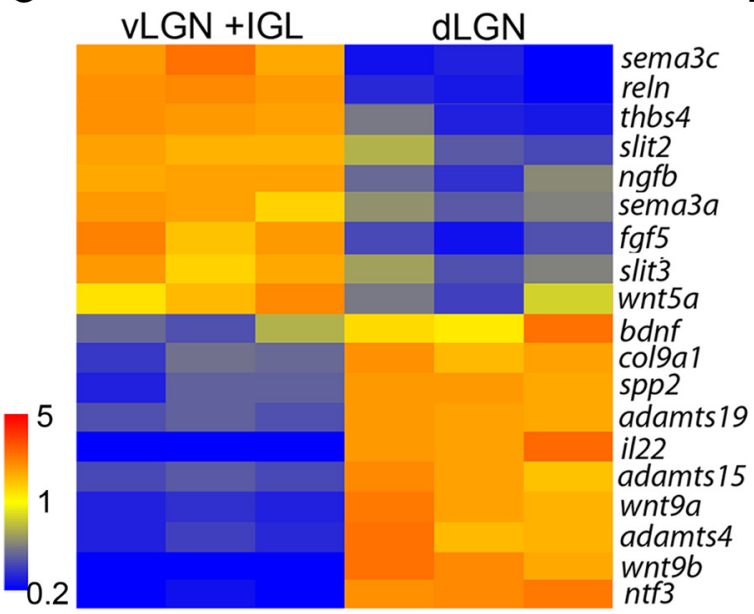

B

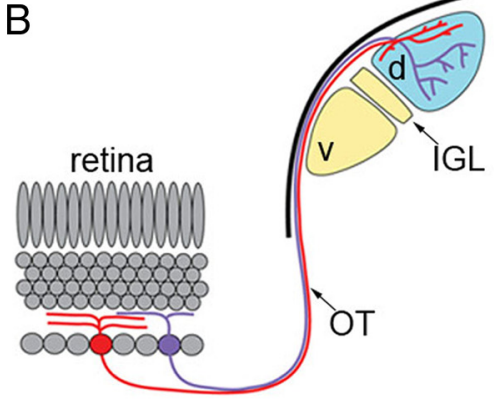

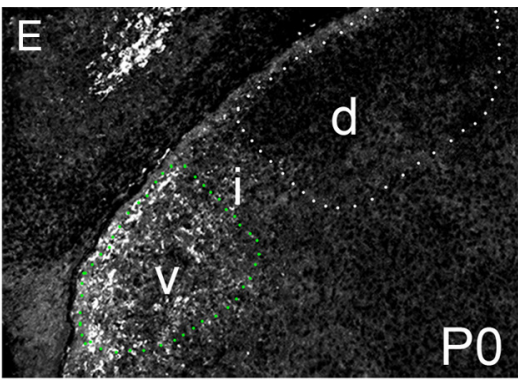
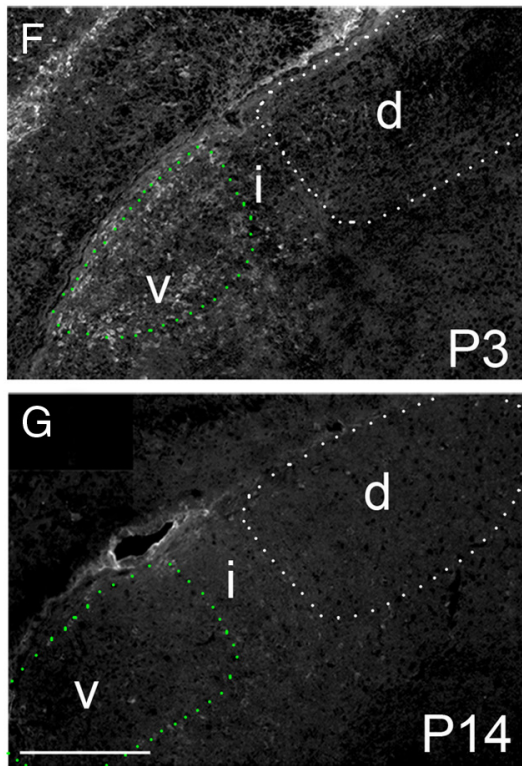

Figure 1. Reelin is selectively expressed in vLGN and IGL. $\boldsymbol{A}, \boldsymbol{B}$, Schematic representation of non-image-forming RGC $(\boldsymbol{A})$ and image-forming RGC ( $\boldsymbol{B})$ axons in $L G N$ subnuclei. $\boldsymbol{C}$, Differentially expressed guidance and targeting cues in either vLGN/IGL or dLGN, displayed in a heat plot and normalized to the mean intensity of each gene. Color scale depicts fold difference between vLGN/IGL and dLGN. RNA was purified from three sets of vLGN/IGL and dLGN samples, each of which was analyzed by Agilent microarray and is represented by a column in the heat plot. Each sample contained tissue pooled from at least five mice. $D$, Developmental regulation of differential gene expression in vLGN/IGL compared with dLGN ( \pm SD) assessed by qPCR. RNA was isolated from five ages. In contrast to other genes, the highest levels of reln mRNA enrichment in $\mathrm{VLGN} / \mathrm{IGL}$ coincided with the arrival of RGC axons. $\boldsymbol{E}-\boldsymbol{G}$, IHC for reelin on coronal sections of mouse thalamus. IHC confirmed the developmental regulation of reelin in vLGN and IGL during periods of retinogeniculate targeting. The dotted green and white lines outline vLGN and dLGN, respectively. Scale bar: (in $\mathbf{G}$ ) $\boldsymbol{E}-\boldsymbol{G}, 300$ $\mu \mathrm{m} . \mathrm{d}, \mathrm{dLGN}$; i, IGL; v, vLGN; OT, optic tract.

mouse LGN-a thalamic nucleus in which classes of imageforming and non-image-forming retinal inputs are segregated into distinct subnuclei. The segregation of class-specific retinal inputs appeared to occur as retinal axons first enter the LGN (supplemental Fig. S1, available at www.jneurosci.org as supplemental material) (Kim et al., 2010), suggesting that molecular cues within LGN subnuclei pattern class-specific retinogeniculate axon targeting. Since there is a paucity of information regarding the factors regulating class-specific targeting of axons in LGN subnuclei, we screened differentially expressed guidance and targeting molecules in either vLGN and IGL (vLGN/IGL) or dLGN. vLGN and IGL were pooled since they receive innervation from similar classes of RGCs (Hattar et al., 2006). RNA was isolated from LGN subnuclei at ages corresponding to the arrival of retinal axons (i.e., P3) (Godement et al., 1984; Jaubert-Miazza et al., 2005) and differentially expressed genes were identified by Agilent microarrays. Numerous genes coding for extracellular matrix molecules and soluble morphogens known to guide axonal growth and targeting were identified as being more than twofold enriched in either vLGN/IGL or dLGN (Fig. 1C). Furthermore, microarray analysis confirmed the differential expression of known transcription factors in either vLGN or dLGN and identified a novel set of LGN subnuclei-specific transcription factors (supplemental Fig. S2, available at www.jneurosci.org as supple- mental material) (Nakagawa and O’Leary, 2001; Jones and Rubenstein, 2004).

In the present study, we focus on molecular cues enriched in and necessary for retinal innervation of vLGN/IGL since all retinogeniculate axons encounter these subnuclei (Fig. $1 A, B$ ). To narrow our list of vLGN/IGL-enriched targeting genes to those likely to contribute specifically to retinogeniculate targeting, we examined the developmental expression of candidate genes at ages corresponding to RGC axon arrival (i.e., P3), arborization, and refinement (P6-P14) (Jaubert-Miazza et al., 2005). The peak expression of one gene-reln-coincided with the arrival of RGC axons (Fig. 1D). Reln encodes the large glycoprotein reelin, which has previously been shown to contribute to both the regulation of neuronal migration (D'Arcangelo et al., 1995) and axonal growth and targeting (Borrell et al., 1999, 2007; Wu et al., 2008). It is noteworthy that microarray analysis not only revealed an approximately sevenfold enrichment in reln $\mathrm{mRNA}$ in vLGN/IGL (compared with dLGN), but also identified an alternatively spliced form of reln mRNA that was more than sixfold enriched in the more ventral LGN subnuclei (data not shown). At P3, reln expression (at both the mRNA and protein level) was highly enriched in vLGN/IGL compared with the dLGN and other retinorecipient nuclei, including the olivary pretectal nucleus (OPN) and suprachiasmatic nucleus (SCN) (Fig. $1 D-F$; supplemental 
Fig. S3, available at www.jneurosci.org as supplemental material). The specificity of reelin IHC at these ages was confirmed by demonstrating a lack of immunoreactivity in LGN of mutant mice lacking reelin (i.e., reln ${ }^{\mathrm{rl} / \mathrm{rl}}$ mutants) (D'Arcangelo et al., 1995) (supplemental Fig. S3, available at www.jneurosci.org as supplemental material). In contrast to its high level of expression from $\mathrm{P} 0$ to $\mathrm{P} 3$, reelin expression in the vLGN/IGL was markedly decreased when RGC axons were no longer entering the LGN (Fig. 1D, G). Based on its developmental expression and previously documented functions, reelin was a prime candidate to contribute to class-specific targeting of RGC axons in the LGN.

Most functions of reelin can be attributed to its binding of very low density lipoprotein receptor (VLDLR) or apolipoprotein E receptor 2 (ApoER2) (Benhayon et al., 2003). For reelin to directly influence retinogeniculate targeting, we hypothesized that classes of RGCs must express reelin receptors. RGCs reside within the innermost layer of the retina, the ganglion cell layer (GCL) (Fig. 2A). In situ hybridization revealed that a small subset of cells within this layer expressed mRNAs encoding either VLDLR or ApoER2 (Fig. $2 B, C$ ).

After binding to VLDLR or ApoER2, reelin induces the activation of Dab1, a cytoplasmic molecule essential for reelin function (Howell et al., 1997; Sheldon et al., 1997). To assess whether Dab1 was also expressed in subsets of RGCs, IHC was performed on P13 wild-type retinal cross-sections. It is noteworthy that previous reports have documented Dabl expression in AII amacrine cells within mouse retina (Rice and Curran, 2000; Rice et al., 2001). Our analysis confirmed expression of Dab1 in amacrine cells within the inner nuclear layer (INL) (Fig. $2 D, E^{\prime}$, arrows) but also revealed a small subset of cells that expressed Dab1 within the GCL (Fig. 2D, $E^{\prime}$, arrowhead). To determine whether these Dab1-expressing cells were RGCs whose axons target vLGN and IGL (i.e., ipRGCs), we colabeled retinal sections for Dabl and Meln, the functional photopigment expressed by ipRGCs. Indeed, these analyses showed that some ipRGCs expressed Dab1 (Fig. 2E).

As previous reports also suggested that Dab1 and reelin were expressed by mutually exclusive cell types within the retina (Rice and Curran, 2000; Rice et al., 2001), we next examined reelin expression in the GCL of P13 wild-type retina. Reelin expression was detected in many, but not all, cells within the GCL (Fig. 2 F). IHC for both reelin and Meln demonstrated that most ipRGCs lacked significant reelin expression $[87.8 \pm 3.2 \%(\mathrm{SD})$ ipRGCs lacked reelin; $N=3$ mice, $E, 15 \mu \mathrm{m} ; \mathbf{D}, \boldsymbol{F}-\mathbf{I}, 20 \mu \mathrm{m}$.
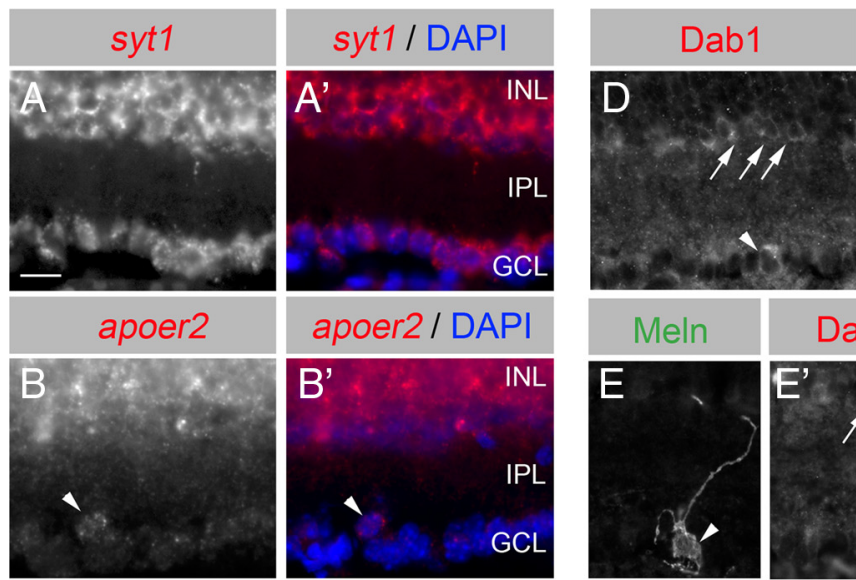
Dab1 / DAPI
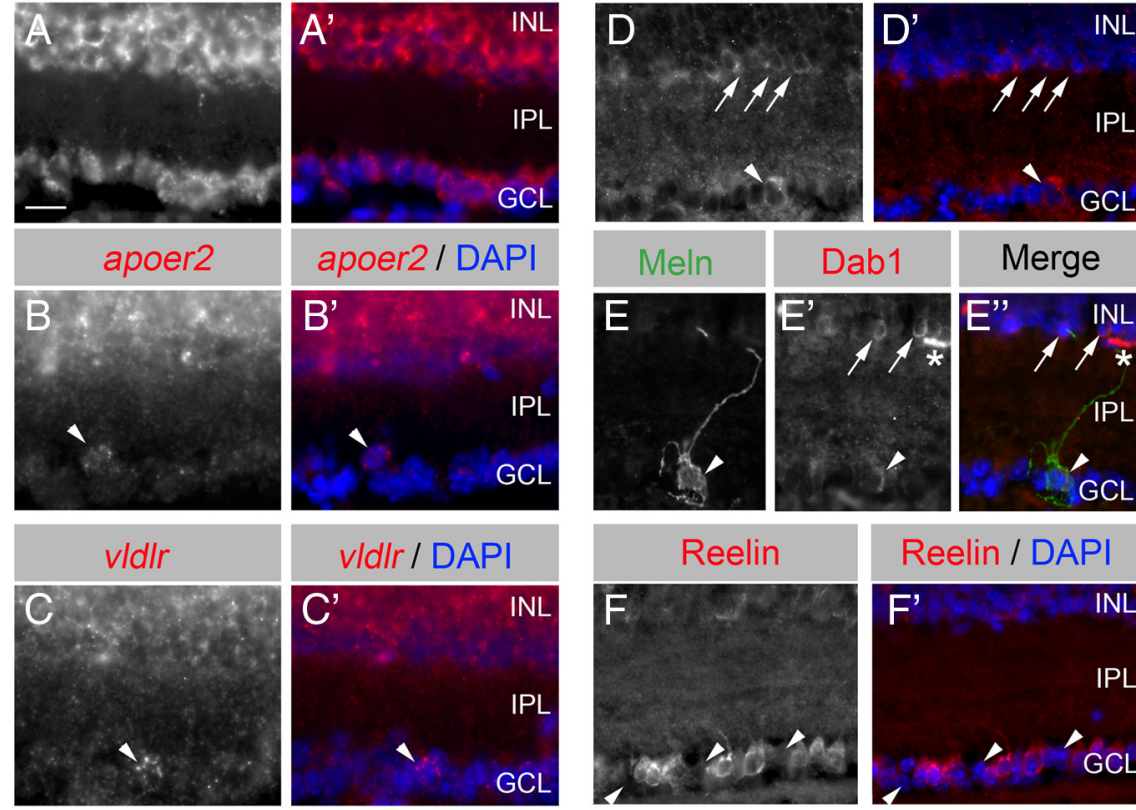

Merge

vldir / DAPI

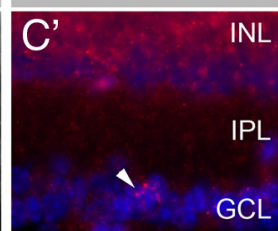

Reelin

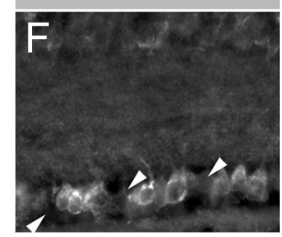

Reelin / DAPI

\section{Reelin}
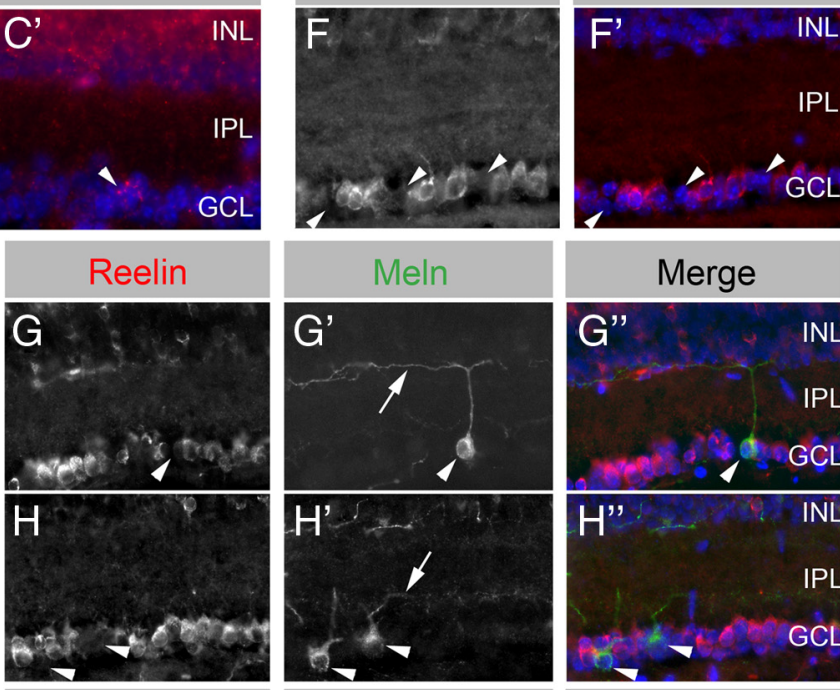

Reelin
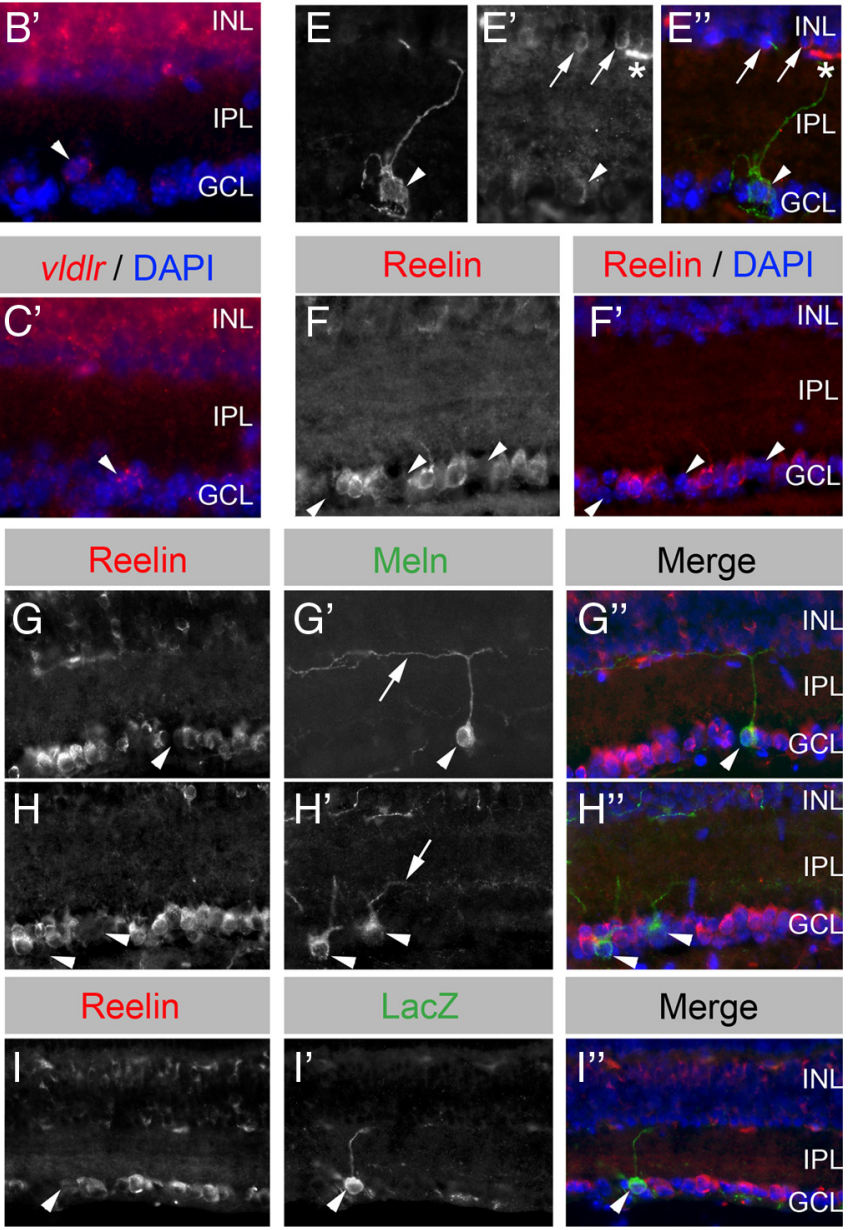

Merge

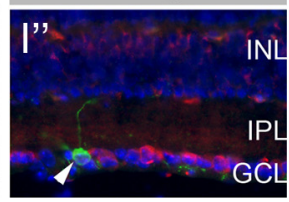

Figure 2. Reelin-signaling components are expressed in the GCL of mouse retina. $A$, ISH for synaptotagmin 1 (syt 1 ) mRNA in P13 wild-type retinal cross-sections demonstrated the distribution of neurons within the GCL. $B, C$, In contrast to the wide distribution of syt1 mRNA, ISH revealed that apoer2 $(\boldsymbol{B})$ and vIdlr ( $\boldsymbol{C}$ ) mRNAs were expressed by small subsets of cells within the GCL (see arrowheads in $\boldsymbol{B}, \boldsymbol{C}$. D, Dab1 $1 \mathrm{HC}$ in P13 wild-type retinal cross-sections confirmed expression of Dab1 by amacrine cells in the INL (arrows) (Rice and Curran, 2000). Sparse labeling of Dab1 was also observed in a subset of cells in the GCL (arrowhead). $\boldsymbol{E}$, Colabeling of Meln and Dab1 in P13 wild-type retinal cross-section. The arrowhead depicts Dab1-expressing ipRGC. The arrows indicate Dab1-expressing amacrine cells. The asterisk denotes nonspecific immunoreactivity. $\boldsymbol{F}$, Reelin IHC in P13 wild-type retinal cross-sections. Many cells within the GCL express reelin; however, the arrowheads indicate cells with no significant reelin expression. $\mathbf{G}, \boldsymbol{H}$, Colabeling of P13 wild-type retinal cross-sections with Meln and reelin antibodies revealed that most ipRGCs do not express reelin (see arrowheads). Meln-expressing RGCs in $\boldsymbol{G}$ and $\boldsymbol{H}$ presumably represent different classes of ipRGCs since there dendrites stratify in different sublaminae of the IPL (see arrows). Both classes of ipRGCs appear to lack reelin expression. $I$, Reelin IHC was performed on P13 retinal cross-sections from opn4-tau-LacZ transgenic mice. M1 ipRGCs were labeled by LacZ IHC. The arrowhead highlights a M1 ipRGC that lacks reelin expression. For all images, nuclei were labeled by DAPI staining. Scale bar: $A-C$,

$n=452$ ipRGCs] (Fig. 2G,H). Melanopsin IHC labeled several classes of ipRGCs (Fig. 2G,H) (Ecker et al., 2010). Classes of ipRGCs whose dendrites stratified in the OFF-sublaminae of the inner plexiform layer (IPL) (Fig. 2G) and those whose dendrites stratified in the ON-sublaminae of the IPL (Fig. $2 \mathrm{H}$ ) both lacked reelin expression. To specifically address reelin expression in M1 
ipRGCs, a class of ipRGC that projects to the vLGN and IGL (Hattar et al., 2006), reelin IHC was performed on retinal sections from transgenic mice in which M1 ipRGCs were selectively labeled with LacZ (opn4-tau-LacZ mice) (Hattar et al., 2002, 2006). Nearly all M1 ipRGCs lacked significant reelin expression [97.9 \pm $0.2 \%$ (SD) M1 ipRGCs lacked reelin expression; $N=2$ mice; $n=$ 194 RGCs] (Fig. 2I). Therefore, our data show that classes of ipRGCs express Dab1 but not reelin. Together, these studies reveal that some RGCs have the machinery to respond to LGNderived reelin.

\section{Reelin and its canonical signaling pathway are necessary for RGC targeting of the vLGN and IGL}

To assess the role of reelin in retinogeniculate targeting, we analyzed retinal projections in $r e n^{\mathrm{rl} / \mathrm{rl}}$ mutants. Intraocular injections of fluorescently conjugated CTB were used to label all RGC axons (Fig. 3A) (Muscat et al., 2003; Jaubert-Miazza et al., 2005). Several striking defects were observed in RGC projections in rel$n^{\mathrm{rl} / \mathrm{rl}}$ mutants. First, the spatial extent of retinal innervation to IGL and vLGN, but not dLGN, was markedly reduced in mutants $\left[45.1 \pm 6.5 \%(\mathrm{SEM})\right.$ reduced extent of innervation in $\mathrm{reln} \mathrm{n}^{\mathrm{rl} / \mathrm{rl}}$ IGL, $n=6$ mice, $p<0.05$ by Student's $t$ test; $16.4 \pm 3.4 \%$ (SEM) reduced extent of innervation in reln $^{\mathrm{rl} / \mathrm{rl}} \mathrm{vLGN}, n=6$ mice, $p<$ 0.05 by Student's $t$ test] (Fig. $3 A, B$; supplemental Fig. S4, available at www.jneurosci.org as supplemental material). Second, numerous misrouted RGC axons were observed in non-retinorecipient thalamic nuclei adjacent to LGN (Fig. $3 B, D$ ). These misrouted axons originated from both contralateral and ipsilateral eyes and exited mutant LGN at either the IGL-dLGN border (Fig. $3 B, D$ ) or from the vLGN (data not shown). Reduced innervation and misrouting of retinal axons were observed as early as P1 (Fig. $3 E, F$ ), suggesting that reelin was necessary for the initial targeting of retinal axons to the vLGN and IGL.

To determine whether reelin required Dab1 function for its role in retinogeniculate targeting, we examined retinal innervation of the LGN in scrambler mutants ( $\left.d a b 1^{\mathrm{scm} / \mathrm{scm}}\right)$, which lack functional Dab1 (Howell et al., 1997; Sheldon et al., 1997). In all dab $1^{\mathrm{scm} / \mathrm{scm}}$ mutants examined $(n=10)$, misrouted RGC axons were observed exiting the LGN medially to enter non-retinorecipient thalamic nuclei (Fig. $4 A-C$ ). In contrast to $r e n^{\mathrm{rl} / \mathrm{rl}} \mathrm{mu}-$ tants, however, only $50 \%$ of $d a b 1^{\mathrm{scm} / \mathrm{scm}}$ mutants displayed sparse retinal arbors in the IGL (Fig. $4 \mathrm{~B}$ ). In mutants in which a striking avoidance of the IGL was not observed, retinal arbors in the mutant IGL appeared disorganized compared with controls (Fig. $4 A, C)$. As was observed in $r e l n^{\mathrm{rl} / \mathrm{rl}}$ mutants, defects in the pattern of IGL innervation were observed as early as P2 (Fig. 4D-F). Together, these findings suggest that reelin signals, at least in part, through Dab1 to pattern the initial formation of retinogeniculate circuits.

In addition to its role in regulating axonal growth and targeting, the reelin-Dab1 signaling pathway has well documented roles in neuronal migration and lamination (D'Arcangelo et al., 1995; Howell et al., 1997; Sheldon et al., 1997). In contrast to other regions of the reln $^{\mathrm{r} / \mathrm{rl}}$ mutant brain, few significant alterations in the cytoarchitecture of mutant LGN have been documented. Our analysis confirmed a relatively normal distribution of neurons (by NeuN IHC) in mutant LGN (Fig. 5A,B). A reduction in the size of the LGN in reln ${ }^{\mathrm{r} / \mathrm{rl}}$ mutants was observed, but this difference was not restricted to the LGN and was presumably attributable to mutants being considerably smaller than littermate controls (Falconer, 1951). One defect that has been reported in $r e n^{\mathrm{rl} / \mathrm{rl}}$ mutant LGN is the presence of aberrant axonal tracts coursing through mutant dLGN (Frost et al., 1986). Axon-

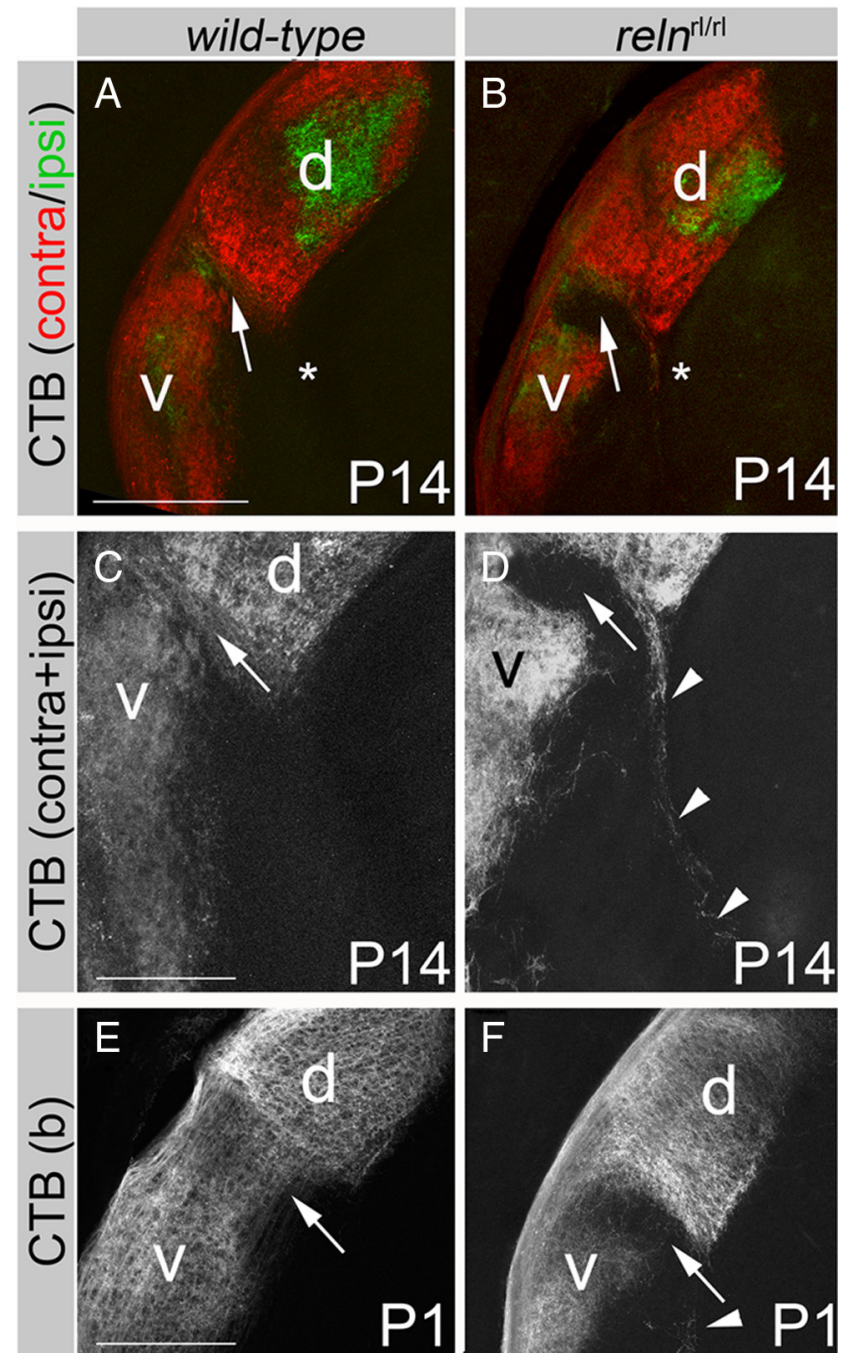

Figure 3. Reelin is necessary for retinogeniculate targeting. $\boldsymbol{A}, \boldsymbol{B}$, Retinogeniculate projections in P14 control $(\boldsymbol{A})$ or reln ${ }^{\mathrm{r} / \mathrm{rl}}$ mutant mice $(\boldsymbol{B})$ assessed by labeling of RGC axons with CTB. The left eyes were injected with Alexa Fluor 594-CTB and right eyes with Alexa Fluor 488-CTB. LGN from right hemispheres are shown. "Contra" denotes retinal arbors originating from cells in the contralateral eye, and "ipsi" denotes those originating from ipsilateral eye. In mutant LGN $(\boldsymbol{B})$, note the near absence of retinal projections in IGL (arrows), the reduced pattern of vLGN innervation, and the presence of mistargeted axons (asterisk). $\boldsymbol{C}$ and $\boldsymbol{D}$ show highmagnification images of areas depicted by asterisks in $\boldsymbol{A}$ and $\boldsymbol{B}$, respectively. Projections from both eyes have been merged into a grayscale image for improved visualization $\left[\right.$ CTB(contra + ipsi)]. $\boldsymbol{E}, \boldsymbol{F}$, Retinogeniculate projections in $\mathrm{P} 1$ control $(\boldsymbol{E})$ or reln ${ }^{\mathrm{r} / \mathrm{rl}}$ mutant mice $(\boldsymbol{F}) . \mathrm{RGC}$ axons from both eyes were labeled with the same fluorescently conjugated form of CTB [CTB(b)]. The arrowhead in $\boldsymbol{F}$ highlights mistargeted RGC axons. The arrows indicate IGL. d, $\mathrm{dLGN}$; v, vLGN. Scale bars: (in $\boldsymbol{A}) \boldsymbol{A}, \boldsymbol{B}, 400 \mu \mathrm{m}$; (in $\boldsymbol{C}) \boldsymbol{C}, \boldsymbol{D}, 200 \mu \mathrm{m}$; (in $\boldsymbol{E}) \boldsymbol{E}, \boldsymbol{F}, 300 \mu \mathrm{m}$.

tracing studies revealed that such misrouted axon tracts did not arise from the retinal axons but instead were formed by corticothalamic axons originating from layer VI cortical neurons (supplemental Fig. S5, available at www.jneurosci.org as supplemental material). The location of these corticothalamic axons in dLGN and the non-retino-recipient portion of vLGN suggest they are unlikely to contribute to the mistargeting of retinal axons in $r e l n^{\mathrm{rl} / \mathrm{rl}}$ mutant LGN.

We next explored whether defects in retinogeniculate targeting resulted indirectly from an abnormal formation of the three LGN subnuclei in absence of reelin. LGN subnuclei are each derived from different embryonic origins (Altman and Bayer, 1989; Botchkina and Morin, 1995); therefore, to examine whether each 
were properly generated in the absence of functional reelin, we assayed the distribution of several known subnuclei-specific markers in $r e l n^{\mathrm{rl} / \mathrm{rl}}$ mutant LGN. The IGL, but not the adjacent vLGN or dLGN, contain an abundance of NPY-expressing relay neurons, as well as glial fibrillary acidic protein (GFAP)-expressing astrocytes (Botchkina and Morin, 1995) (Fig. 5A, C). Many interneurons and projection neurons within the vLGN and IGL express Gad65 (Morin et al., 1992; Moore and Speh, 1993) (Fig. 5E). Relay neurons within the vLGN and dLGN, but not IGL, contain an isoform of neurofilament recognized by the antibody SMI32 (Fig. 5I) (Jaubert-Miazza et al., 2005). In addition to these known markers, we identified two novel subnuclei-specific markers. Neurons within the vLGN, but not the IGL or dLGN, express synaptotagmin 2 (syt2) mRNA (Fig. 5G), whereas only neurons within the dLGN express "a disintegrin and metalloproteinase with a thrombospondin type 1 motif member 15" (adamts15) mRNA (Fig. 5K). Immunostaining and in situ hybridization for each of these markers revealed that the three LGN subnuclei form appropriately and remain distinct in $\mathrm{reln}^{\mathrm{rl} / \mathrm{rl}}$ mutants (Fig. 5). Importantly, NPY-expressing IGL relay neurons, which are the targets of RGC axons (Thankachan and Rusak, 2005), remain confined to the IGL and were not dispersed into inappropriate thalamic regions (Fig. $5 A, B, K, L$ ). Together, these data suggest that misrouted axons in $\mathrm{reln}{ }^{\mathrm{r} l / \mathrm{rl}}$ mutants are in fact mistargeted rather than correctly targeted to neurons that have migrated into inappropriate regions of thalamus.

\section{Reelin is required for the targeting of ipRGCs in the LGN}

Defects in the pattern of retinal innervation to reln $^{\mathrm{rl} / \mathrm{rl}} \mathrm{vLGN}$ and IGL led us to hypothesize that reelin was essential for retinogeniculate targeting by ipRGCs. To ensure that defects in vLGN and IGL targeting were not consequences of intraretinal defects, we examined the distribution and morphology of ipRGCs in rel$n^{\mathrm{rl} / \mathrm{rl}}$ mutant retina. Whole-mount Meln IHC revealed that the distribution and morphology of ipRGCs were indistinguishable between mutant or control retinas (Fig. 6A, $B$; supplemental Fig. S5 $A$, available at www.jneurosci.org as supplemental material). Additionally, numbers of Meln-immunoreactive ipRGCs appeared similar in whole-mount preparations of P12 mutant and control retinas [controls contained $42.4 \pm 7.9$ (SD) ipRGCs per field, $N=11$ retinas, $n=86$ fields; mutants contained $41.3 \pm$ 9.6 (SD) ipRGCs per field, $N=12$ retinas, $n=101$ fields]. Importantly, the observed defects in retinogeniculate targeting were also not attributable to an inability of mutant ipRGCs to endocytose or anterogradely transport CTB (Fig. $6 A^{\prime}, B^{\prime}, C-F$ ). Thus, defects in RGC innervation of vLGN and IGL did not appear to arise from intraretinal defects in ipRGCs.

We also assessed whether ipRGC axons correctly targeted other appropriate retino-recipient nuclei in $\mathrm{reln}^{\mathrm{rl} / \mathrm{rl}}$ mutants. Classes of ipRGCs innervate the OPN and SCN (Muscat et al., 2003; Hattar et al., 2006; Ecker et al., 2010). CTB labeling in reln ${ }^{\mathrm{r} l / \mathrm{rl}}$ mutants revealed normal patterns of retinal innervation to these nuclei (Fig. $6 C-F$ ). Furthermore, reln ${ }^{\mathrm{rl} / \mathrm{rl}}$ mutants retained rudimentary PLRs, behaviors that depend on ipRGC innervation of the OPN (Fig. 6G,H) (Güler et al., 2008). Therefore, in the absence of reelin ipRGCs were not only morphologically normal but generated axons capable of targeting some appropriate retino-recipient nuclei and retained rudimentary function.

To directly assess whether ipRGC axons were misrouted in the $r e n^{\mathrm{r} 1 / \mathrm{rl}}$ LGN, mutants were crossed with opn4-tau-LacZ mice, since M1 ipRGCs are labeled in this line and M1 ipRGCs densely innervate vLGN and IGL (Fig. 7A) (Hattar et al., 2006; Ecker et al., 2010). In P11 reln ${ }^{\mathrm{r} / \mathrm{rl}} ;$ opn4-tau-LacZ mutants, several defects were observed in the pattern of ipRGC projections to vLGN and IGL. First, M1 ipRGC projections avoided central regions of mutant IGL and instead formed a dense, narrow network of fibers adjacent to the ventral aspect of dLGN (Fig. 7B). Second, a large cohort of ipRGC axons invaded ventromedial regions of dLGN (Fig. $7 B^{\prime}, D^{\prime}$, arrows). The area of dLGN occupied by misrouted M1 ipRGC axons was measured in P21 mutants and controls. Although few M1 ipRGC arbors were observed in control dLGN, nearly $20 \%$ of the area of the $\mathrm{reln}^{\mathrm{r} / \mathrm{rl}}$ mutant $\mathrm{dLGN}$ contained LacZ-expressing ipRGC arbors [2.9 $\pm 1.1 \%$ (SEM) of the area of control dLGN contained M1 ipRGC arbors versus $19.2 \pm 2.9 \%$ (SEM) in reln ${ }^{\mathrm{rl} / \mathrm{rl}}$ mutant dLGN; $N=6$; $p<0.05$ by Student's $t$ test]. Last, the majority of retinal axons misrouted out of the IGL or vLGN contained LacZ, revealing they originated from M1 ipRGCs (Fig. $7 B, C$ ).

We next examined the role of Dab1 in the correct targeting of M1 ipRGC axons. Scrambler mutants ( $\left.d a b 1^{\mathrm{scm} / \mathrm{scm}}\right)$ were crossed 

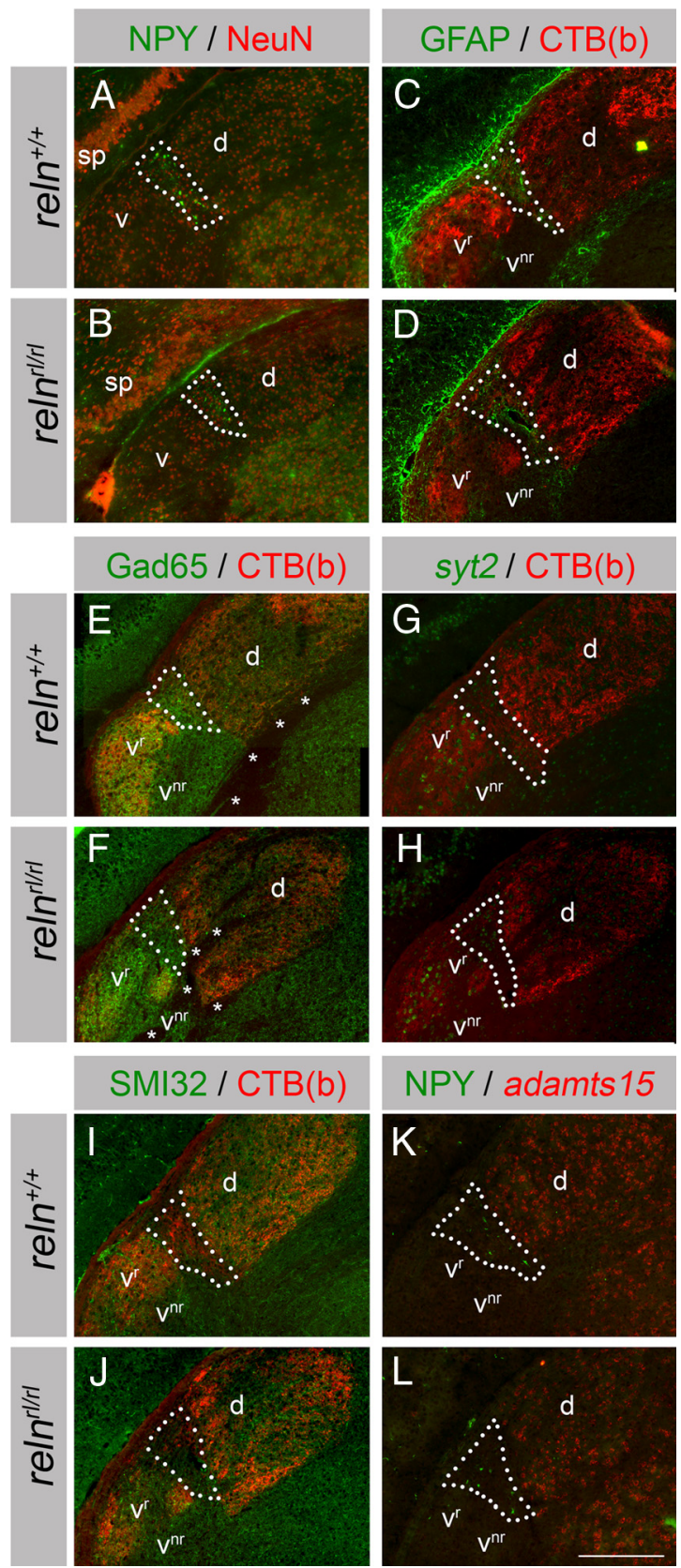

Figure 5. LGN subnuclei are present, remain distinct, and contain appropriate neural populations in the absence of functional reelin. IHC and ISH were performed on coronal sections of P14-P21 reln ${ }^{r / r l}$ mutant or littermate control brains with subnuclei- or neuron-specific markers. $A, B$, NeuN and NPY IHC in P14 reln ${ }^{r / r l}$ mutant or control LGN. NPY remained properly restricted to the IGL in the absence of reelin. $C, D, G F A P I H C$ demonstrated the presence and confinement of astrocytes in P21 control and mutant IGL. $\boldsymbol{E}, \boldsymbol{F}, \mathrm{IHC}$ revealed a normal distribution of Gad65 in P21 reln ${ }^{\mathrm{rl} / \mathrm{rl}}$ mutant vLGN and IGL. $\mathbf{G}, \boldsymbol{H}$, ISH revealed a confinement of syt2-expressing neurons in vLGN of P21 mutants and controls. I, J, SMI32 immunoreactivity was appropriately enriched in $\mathrm{VLGN}$ and dLGN, but absent from IGL in P21 mutants and controls. $K$, L, Adamts 15 mRNA, assessed by ISH, was correctly localized in P14 mutant and control dLGN. Sections were costained with NPY antibodies to demonstrate the location of the IGL. Note that NPY-positive cells did not invade dLGN in the absence of functional reelin. In $\boldsymbol{C}-\boldsymbol{J}$, retinal axons were anterogradely labeled with CTB (CTB[b]). In each image, the IGL is encircled by white dots. d, dLGN; $v$, $v L G N ; v_{r}$, retino-recipient portion of $v L G N ; v_{n r}$, non-retino-recipient portion of vLGN. The asterisks denote corticothalamic axon tracts. Single-color panels for each merged image can be found in supplemental Figure 6 (available at www.jneurosci.org as supplemental material). Scale bar, $400 \mu \mathrm{m}$.
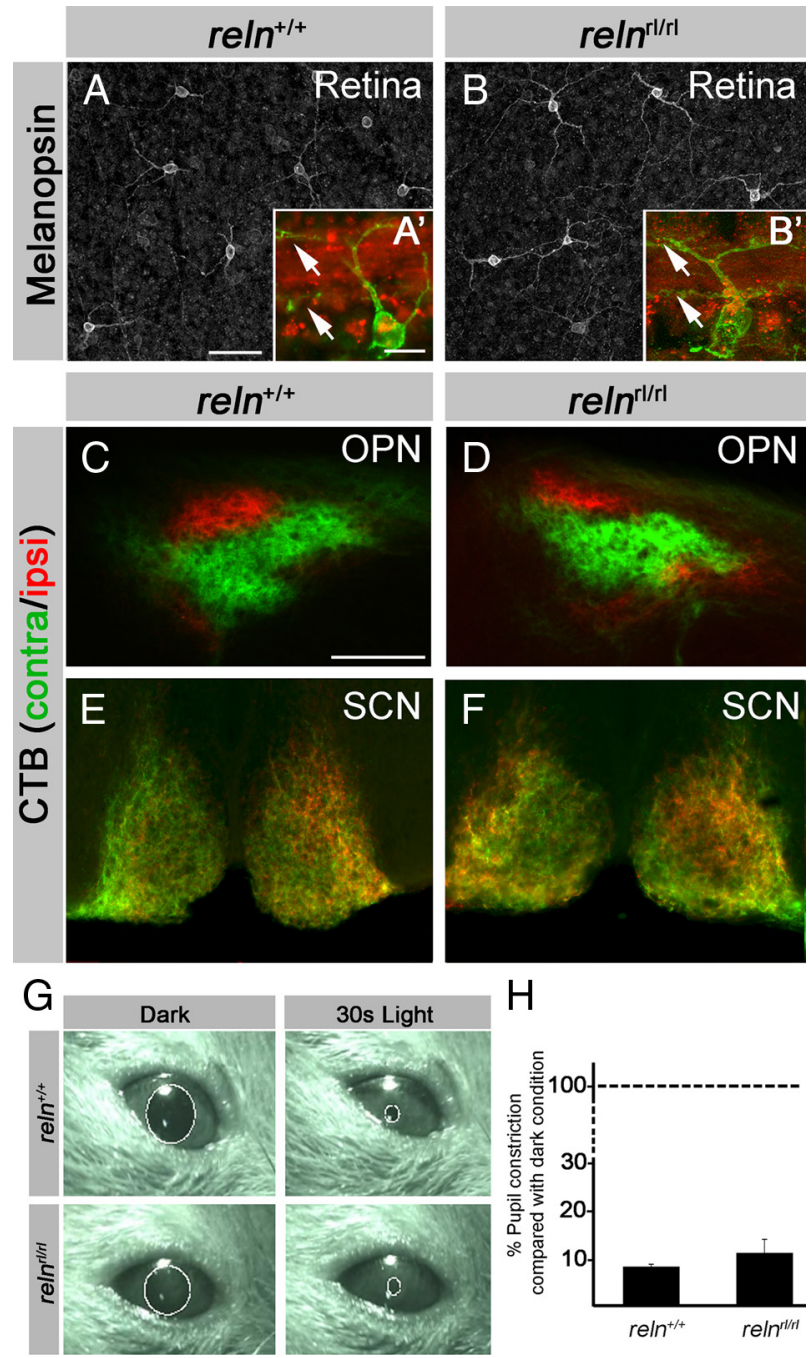

$\mathrm{H}$
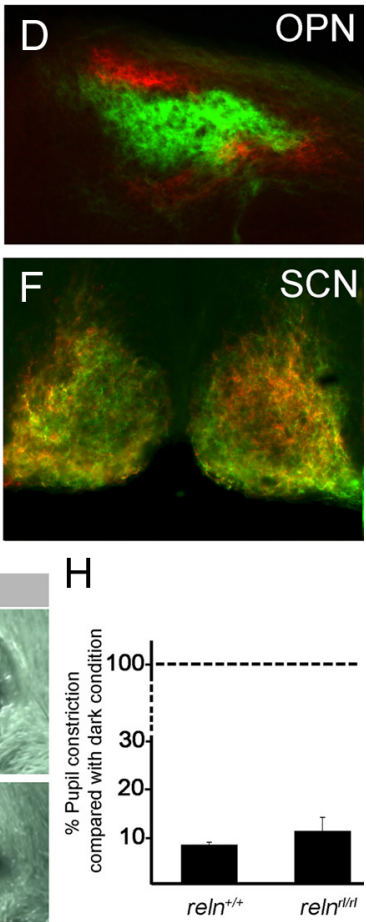

Figure 6. Reelin is not required for the generation or development of non-image-forming $\mathrm{RGCS}$, nor their projections to non-LGN retino-recipient nuclei. $\boldsymbol{A}, \boldsymbol{B}$, Non-image-forming ipRGCS were labeled by Meln IHC in P12 control and reln ${ }^{r / 1 / r}$ mutant retinal whole mounts. The distribution and morphology of ipRGCs appeared similar in controls and mutants. $\boldsymbol{A}^{\prime}, \boldsymbol{B}^{\prime}$, Dendritic stratification of melanopsin-immunolabeled ipRGCs (green) in OFF- and ON-sublaminae of the IPL (arrows) appeared similar in cross-sections of P12 re/ $n^{r / / r l}$ mutant and littermate control retinas. Moreover, mutant and control ipRGCs (green) demonstrated equal capacities to endocytose CTB (red). C, D, Retinal projection to other retino-recipient nuclei innervated by ipRGC appear normal in the absence of functional reelin. $\operatorname{RGC}$ projections to the $\mathrm{OPN}(\boldsymbol{C}, \boldsymbol{D})$ and $\operatorname{SCN}(\boldsymbol{E}$, F) were assessed by injections of different fluorescently conjugated CTB into each eye of P12 controls and mutants. The patterns of innervation of these nuclei were indistinguishable in mutants or controls. $\mathbf{G}, \boldsymbol{H}$, PLRs remained present in reln ${ }^{\mathrm{rl} / \mathrm{rl}}$ mutants. P14 mutant and control mice ( $n=3$ each) were dark adapted for $>1 \mathrm{~h}$ and then exposed to a $30 \mathrm{~s} 1.7 \mathrm{~mW} / \mathrm{cm}^{2}$ light. Pupil constriction was captured on video, and images were used to measure pupil size ( $\mathbf{G}$, white circles) before the onset of light and after $30 \mathrm{~s}$ of light. In $\boldsymbol{H}$, the percentage pupil constriction after light exposure was calculated by comparing to pupil size during dark adaptation. Scale bars: (in $\boldsymbol{A}) \boldsymbol{A}, \boldsymbol{B}, 100 \mu \mathrm{m}$; (in $\boldsymbol{A}^{\prime}$ ) $\boldsymbol{A}^{\prime}, \boldsymbol{B}^{\prime}, 25 \mu \mathrm{m}$; (in $\left.\boldsymbol{C}\right) \boldsymbol{C}-\boldsymbol{F}, 200 \mu \mathrm{m}$.

to the opn4-tau-LacZ mice described above. In the absence of Dab1, M1 ipRGCs invaded non-retino-recipient thalamic regions and occupied a statistically significant area of mutant dLGN $\left[13.0 \pm 1.5 \%(\mathrm{SEM})\right.$ of $d a b 1^{\mathrm{scm} / \mathrm{scm}}$ mutant dLGN contained M1 ipRGC arbors; $N=6 ; p<0.05$ by Student's $t$ test]. Analyses in both reln ${ }^{\mathrm{rl} / \mathrm{rl}} ;$ opn4-tau-LacZ and in dab $1^{\mathrm{scm} / \mathrm{scm}} ;$ opn4tau-LacZ mice also demonstrated that M1 ipRGC projections to SCN and OPN were unaffected by the absence of functional reelin (supplemental Fig. S6, available at www.jneurosci.org as supplemental material) (data not shown) (Hattar et al., 2006; Ecker et 
al., 2010). These findings identify, for the first time, that at least one class of nonimage-forming retinal axons require reelin and Dab1 for targeting appropriate LGN subnuclei.

Reelin is not required for subnucleispecific targeting of dLGN-projecting retinal axons

We next tested whether image-forming classes of RGCs require reelin for appropriately targeting dLGN. For this, we used three approaches_labeling retinal terminals, labeling dLGN-projecting axons, and colabeling misrouted retinal axons with CTB and cell-specific markers of dLGN-projecting axons. To label axon terminals from image-forming RGCs, we examined the distribution of VGLUT2, a synaptic component enriched in dLGN-projecting retinal axons (Fig. 8A) (Land et al., 2004). In $r e l{ }^{\mathrm{rl} / \mathrm{rl}}$ mutant LGN, the density and distribution of VGLUT2 immunoreactivity appeared remarkably similar to controls, suggesting that image-forming, dLGNprojecting RGC axons were not misrouted into vLGN or IGL in the absence of functional reelin (Fig. $8 \mathrm{~B}$ ). Second, calretininexpressing dLGN-projecting retinal axons (Lüth et al., 1993; Huberman et al., 2008b) were labeled in mutants and controls by IHC. As in control LGN, calretinincontaining retinal axons were excluded from vLGN and IGL in reln ${ }^{\mathrm{rl} / \mathrm{rl}}$ mutant LGN (Fig. 8C,D). VGLUT2 or calretinin immunoreactivity alone could not reveal whether axons misrouted into nonretino-recipient thalamic nuclei in reln ${ }^{\mathrm{rl} / \mathrm{rl}}$ mutants originated from image-forming classes of RGC. To test whether any mistargeted RGC axons belonged to image-forming classes of RGCs, axons in reln ${ }^{\mathrm{rl} / \mathrm{rl}}$ mutant thalamus were colabeled by calretinin IHC and CTB. No misrouted CTB-labeled RGC axons were observed to contain calretinin in mutant thalamus (Fig. $8 E, F$ ). Together, these experiments demonstrate that classes of image-forming RGC axons correctly target dLGN in the absence of functional reelin.

\section{Reelin is not required for subnuclei-specific targeting of layer} VI corticothalamic axons

In addition to retinal axons, cortical axons differentially target LGN subnuclei (Jacobs et al., 2007). Cortical neurons in layer VI project axons that course through the internal capsule and around vLGN and IGL to selectively innervate dLGN (Fig. 9A). Despite coursing around vLGN and IGL, these corticothalamic axons do not enter or arborize in ventral LGN subnuclei. To address whether cortical axons required reelin for normal LGN targeting, reln ${ }^{\mathrm{rl} / \mathrm{rl}}$ mutants were crossed to golli-tau-GFP transgenic mice, in which layer VI neurons were selectively labeled with GFP (Jacobs et al., 2007). Corticothalamic axons from layer VI neurons
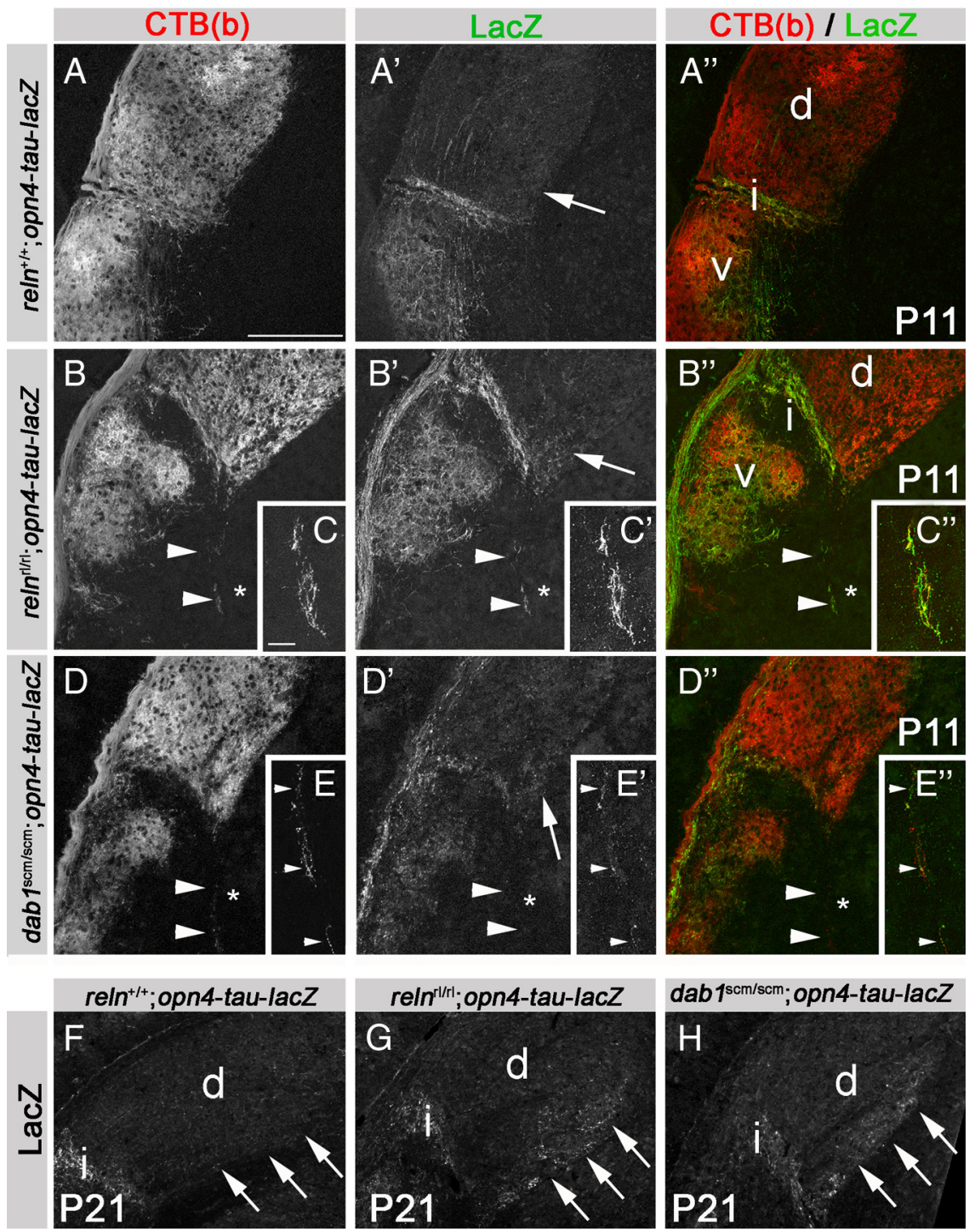

Figure 7. Non-image-forming ipRGCs require reelin and Dab1 for retinogeniculate targeting. $\boldsymbol{A}-\boldsymbol{E}$, IpRGC projections in P11 by LacZ IHC (green). All RGC axons were labeled by binocular injection of CTB (CTB[b]; red). The arrowheads in $\boldsymbol{B}-\boldsymbol{E}$ indicate mistargeted RGC axons containing LacZ. The arrow in $\boldsymbol{B}^{\prime}$ and $\boldsymbol{D}^{\prime}$ highlight misrouted LacZ-containing M1 ipRGC axons invading ans in $\boldsymbol{B}$ and $\boldsymbol{D}$. Note that LacZ immunoreactivity is reduced in $d a b 1^{\mathrm{scm} / \mathrm{scm}} ; 0$ opn4-tau-LacZ ${ }^{+/-}$mutants (D) compared with within dLGN in re/n ${ }^{\mathrm{r} / \mathrm{rl}} ;$;opn4-tau-LacZ and dab $1^{\mathrm{scm} / \mathrm{scm}} ;$ opn4-tau-LacZ mutants, but not controls (compare arrows in $\boldsymbol{F}$-H). Scale bars: (in $\boldsymbol{A}) \boldsymbol{A}, \boldsymbol{B}, \boldsymbol{D}, \boldsymbol{F}-\boldsymbol{H}, 300 \mu \mathrm{m}$; (in $\boldsymbol{C}$, $, \boldsymbol{E}, 25 \mu \mathrm{m}$.

avoided vLGN and IGL and selectively innervate dLGN in controls (Fig. 9B,D). As expected, disruptions in cortical layering were observed in reln $^{\mathrm{rl} / \mathrm{rl}} ;$ golli-tau-GFP mutant mice (Fig. 9C). As described above, axons from layer VI cortical neurons also displayed fasciculation defects and took altered courses through the internal division of the vLGN, a region that does not receive retinal input (Harrington, 1997). Yet despite defects in neuronal position and axonal trajectory, layer VI corticothalamic axons correctly avoided the IGL and retino-recipient portion of the vLGN and arborized only in the dLGN of reln ${ }^{\mathrm{rl} / \mathrm{rl}}$; golli-tau-GFP mutants (Fig. 9E). We draw two important conclusions from these findings: neither reelin nor neuronal position are critical determinants for layer VI corticothalamic axons to correctly avoid vLGN and IGL and specifically target dLGN. 


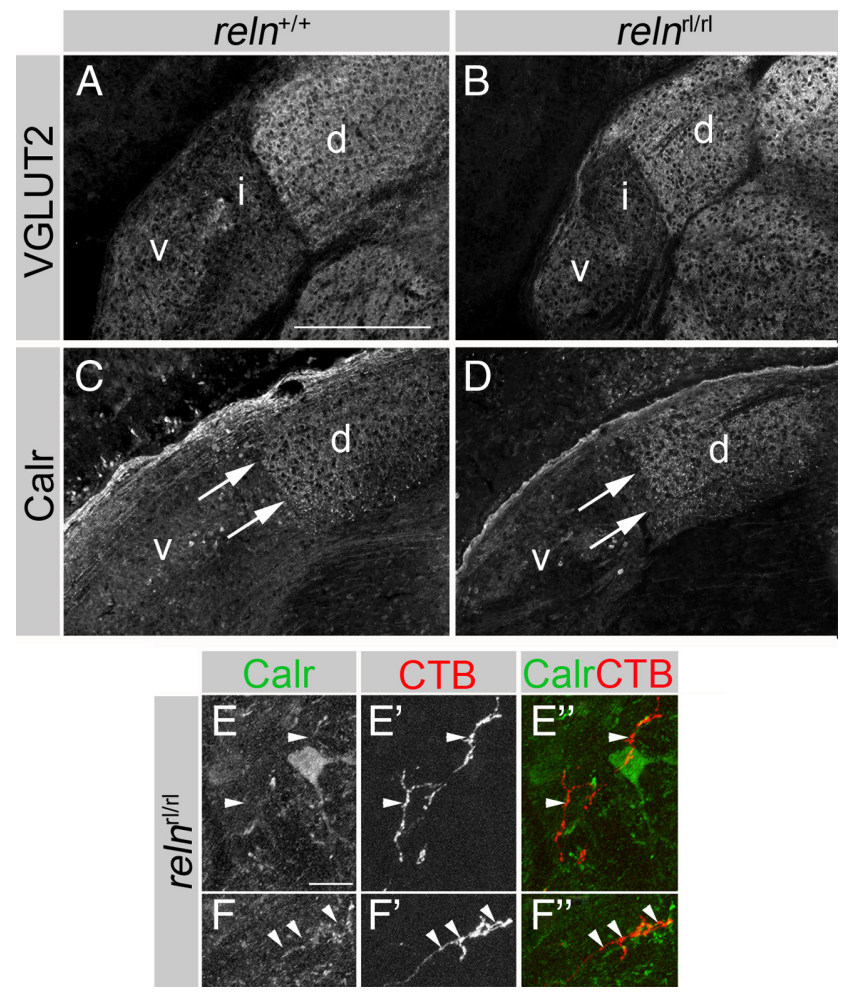

Figure 8. Reelin is dispensable for dLGN targeting by axons derived from image-forming RGCS. $A$, $B$, VGLUT2-immunolabeled dLGN-projecting retinal terminals appear similar in P12 controls $(A)$ and reln ${ }^{n / / r}$ mutants $(\boldsymbol{B}) . \boldsymbol{C}, \boldsymbol{D}$, Calretinin (Calr)-immunolabeled RGC axons appeared similarly confined to $\mathrm{dLGN}$ in P12 control (C) and reln ${ }^{\mathrm{r} / \mathrm{l} / 1}$ mutant (D) LGN. The arrows indicate abrupt border of Calrcontaining arbors at the dLGN-IGL border. $E$, F, Misrouted (TB-labeled RGC axons in adjacent nonretino-recipient thalamic nuclei do not contain Calr in P14 reln ${ }^{\mathrm{H} / \mathrm{ll}}$ mutants (see arrowheads). Scale bars: (in $\boldsymbol{A}) \boldsymbol{A}-\boldsymbol{D}, 300 \mu \mathrm{m}$; (in $\boldsymbol{E}) \boldsymbol{E}, \boldsymbol{F}, 15 \mu \mathrm{m}$.

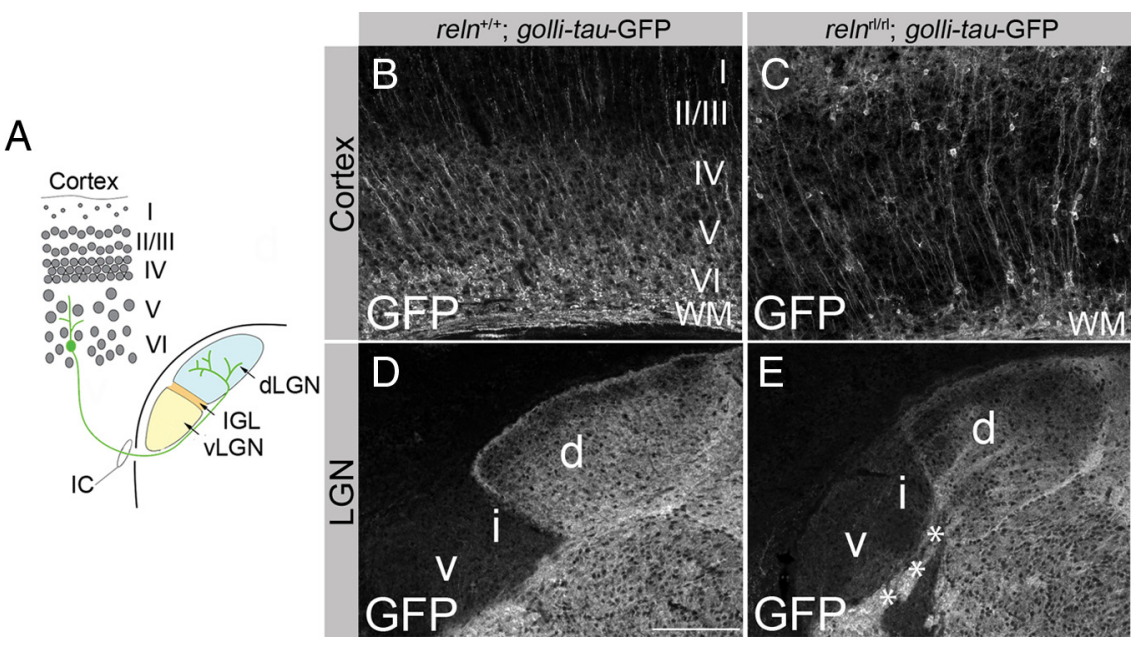

Figure 9. Reelin is dispensable for dLGN targeting by corticothalamic axons. A, Schematic demonstrating layer VI cortical axons pass through the internal capsule (IC), bypass VLGN/IGL, and selectively innervate dLGN. B, C, Layer VI cortical neurons were labeled by GFP IHC in P12 reln ${ }^{+/+}$; golli-tau-GFP controls (B) or reln ${ }^{\text {rl/rll }}$;olli-tau-GFP mutants (C). Normal cortical layering and the location of white matter tracts (WM) are depicted in $\boldsymbol{B}$. Layer VI cortical neurons and their projections in transgenic mice were labeled by GFP IHC. D, E, Although corticothalamic axons have an altered course in rel/ ${ }^{r / 1 / 1}$; golli-tau-GFP mutants (see asterisks in E), they selectively target dLGN as in reln ${ }^{+/+}$; golli-tau-GFP controls. d, dLGN; v, vLGN; i, IGL. Scale bar, $300 \mu \mathrm{m}$.

\section{Discussion}

Identifying the mechanisms that underlie class-specific targeting of axons to appropriate regions of the brain is critical for our understanding of how complex neural circuits-and their asso- ciated behaviors-are established. Although the visual system has long been a useful model system in studying the initial establishment and subsequent refinement of neural circuits, the vast number of RGCs and the difficulty in labeling individual classes of RGCs have hampered investigations aimed at elucidating the mechanisms of class-specific targeting. Here, rather than focusing on identifying the mechanisms that drive the targeting of a single class of RGCs, we focused on identifying cues that may drive the segregation of RGC axons that convey either imageforming visual input or non-image-forming visual input. In doing so, we identified a plethora of candidate targeting molecules differentially expressed in LGN subnuclei. Analysis of one of these cues-reelin — revealed for the first time a molecule necessary for class-specific retinogeniculate targeting.

\section{Differential expression of targeting cues in LGN subnuclei}

To elucidate some of the mechanisms contributing to classspecific axonal targeting, we examined the development of specific connections between functionally distinct classes of RGCs and different LGN subnuclei. Two broad mechanisms have been proposed to generate such specificity: (1) class-specific retinal arbors could initially be diffusely spread throughout the entire LGN and then be refined to appropriate subnuclei based on functional activity; (2) the initial projections of axons originating from a single class of RGCs could be programmed to innervate only appropriate target regions. To distinguish between these possibilities in the LGN, we examined the development of a set of class-specific retinal projections that express Calr and whose arbors are confined to the dLGN of adult rodents (Lüth et al., 1993; Huberman et al., 2008b). As Calr-containing RGC axons initially entered the LGN, they bypassed the vLGN and IGL and arborized selectively in dLGN (supplemental Fig. S1, available at www. jneurosci.org as supplemental material) (Kim et al., 2010). This suggested that the targeting of classspecific retinal axons to LGN subnuclei was driven by specific targeting cues and not by activity-dependent refinement. By comparing mRNA transcripts in either vLGN/IGL or dLGN, we identified numerous families of molecules known to direct the growth, guidance, and targeting of axons that were differentially expressed in LGN subnuclei. These included, but were not limited to, semaphorins, neurotrophins, growth factors, slits, Wnts, and ECM molecules (i.e., reelin, thrombospondin 4, collagen IX, and several members of ADAMTS family) (Huber et al., 2003; Fox, 2008; Hamel et al., 2008; Sanes and Yamagata, 2009; Huberman et al., 2010).

Based on its high enrichment in perinatal vLGN/IGL (approximately sevenfold enriched in vLGN/IGL compared with dLGN by microarray) (Fig. 1C) and its developmental regulation (Fig. $1 D-G$ ), we focused here on the role of reelin in class-specific retinogeniculate targeting. Our results demonstrate that reelin and its canonical signaling pathway are critical for the targeting of ipRGC axons to the vLGN and IGL. However, other aspects of class-specific retinogeniculate (and corticothalamic) targeting were unaffected by the absence of functional reelin. For example, 
dLGN-projecting retinal and cortical axons did not require reelin to correctly avoid vLGN or IGL as they coursed around these regions. Moreover, based on the abundance of RGC axons in $r l^{\mathrm{r} l / \mathrm{rl}}$ mutant $\mathrm{vLGN}$, it appears likely that classes of vLGNprojecting RGC axons exist that do not require reelin for targeting. Here, we have demonstrated that M1 ipRGCs require reelin, but several other classes of ipRGCs exist that target vLGN (Ecker et al., 2010); it remains unclear whether these classes require reelin. Since reelin-independent aspects of class-specific retinogeniculate targeting exist, it is possible-perhaps likely-that other guidance and targeting cues identified in our screens may contribute to these aspects of retinogeniculate targeting. Not only have many of the identified families of candidate cues been demonstrated to contribute to axonal guidance and targeting, but many have known effects on RGC axons. Neurotrophins and FGFs affect the terminal branching and targeting of retinal axons (Cohen-Cory and Fraser, 1994, 1995; McFarlane et al., 1995, 1996; Inoue and Sanes, 1997; Webber et al., 2003, 2005). Wnts mediate medial-lateral topographic mapping of retinal projections to the chick tectum (Schmitt et al., 2006). Slits and semaphorins both inhibit or repel the growth of retinal axons (Niclou et al., 2000; Steinbach et al., 2002). Analysis of these molecules in class-specific targeting of the LGN clearly warrants future attention.

\section{The role of reelin in class-specific retinogeniculate targeting}

In the present study we specifically explored what roles the large extracellular glycoprotein reelin may contribute to retinogeniculate targeting. Previous studies have defined roles for reelin in the targeting of specific classes of axons in the mammalian hippocampus. Layer-specific hippocampal targeting of axons from the entorhinal cortex (EC), but not targeting of commissural axons, requires target-derived reelin (Del Río et al., 1997; Borrell et al., 1999). In vitro assays further demonstrated that such roles for reelin in entorhino-hippocampal connections are attributable, at least in part, to direct roles of reelin on neurons (Borrell et al., 2007). Classes of neurons that are responsive to reelin express Dab1 (Borrell et al., 2007) — an adaptor molecule that is central to the canonical reelin signaling cascade (Howell et al., 1997; Sheldon et al., 1997; Rice et al., 1998). Like reelin, Dab1 expression is essential for layer-specific entorhino-hippocampal connections (Borrell et al., 2007). However, since both reelin and Dab1 are necessary for cortical and hippocampal lamination (Howell et al., 1997; Sheldon et al., 1997), defects observed in hippocampal targeting in mutants lacking either reelin or Dab1 could arise from the correct targeting of cells that have been misplaced. But organotypic cocultures of Dab1-deficient EC with wild-type hippocampi demonstrated that axons from EC neurons lacking Dabl are indeed mistargeted regardless of the position of appropriate partner neurons (Borrell et al., 2007). Together, these studies demonstrate that reelin and its canonical signaling pathway are directly necessary for the formation of class-specific hippocampal connections. Likewise, it has also been reported that reelin and Dab1 are required for synaptic targeting in the retina (Rice et al., 2001).

On the basis of the above-described studies and the spatiotemporal expression of reelin in the LGN, reelin initially appeared to be a prime candidate capable of influencing class-specific retinogeniculate targeting. Our data now provide compelling evidence supporting this: reelin is necessary for the targeting of at least one class of RGC axons (M1 ipRGC axons) to vLGN and IGL. In addition to those defects we report here for M1 ipRGCs, it is noteworthy that defects in the targeting of retinal axons to supe- rior colliculus (SC) have also been described previously in reln ${ }^{\mathrm{rl} / \mathrm{rl}}$ mutants (Frost et al., 1986; Baba et al., 2007). However, our data suggest that the mechanisms responsible for retinogeniculate and retinocollicular targeting defects are different. Defects in neuronal lamination precede the arrival of RGC axons in mutant SC (Baba et al., 2007), suggesting axonal targeting defects arise (at least in part) as a result of abnormal SC cytoarchitecture. In contrast, our data strongly suggest that defects in retinogeniculate targeting in $r e l n^{\mathrm{rl} / \mathrm{rl}}$ mutants do not arise from LGN disorganization or an absence of appropriate target neurons in IGL or vLGN. Based on the presence of mistargeted M1 ipRGC axons in mutant dLGN and non-retino-recipient thalamus_-regions lacking their appropriate target neurons (e.g., NPY-expressing neurons) in both controls and mutants-our data suggest that these ipRGC axons are indeed mistargeted and not merely targeting correct partners that are misplaced into inappropriate LGN subnuclei in the absence of functional reelin. In support of this hypothesis, we found that like EC neurons (Borrell et al., 2007) some ipRGCs express Dab1 and may therefore be capable of directly responding to LGN-derived reelin. We further discovered that, similar to layer-specific entorhino-hippocampal connections, the formation of retinogeniculate connections required Dab1. As our findings strongly parallel those supporting the role of reelin in entorhino-hippocampal targeting, we suspect a more direct role for reelin in class-specific retinogeniculate targeting. Of course, it remains possible that multiple mechanisms are responsible for the defects observed in the targeting of $\mathrm{reln}^{\mathrm{rl} / \mathrm{rl}} \mathrm{LGN}$. This possibility may shed light on why misrouted RGC axons were observed in all of the $d a b 1^{\mathrm{scm} / \mathrm{scm}}$ mutant LGN examined, but only $50 \%$ of these exhibited a lack of RGC axons within the putative IGL.

\section{References}

Altman J, Bayer SA (1989) Development of the rat thalamus: VI. The posterior lobule of the thalamic neuroepithelium and the time and site of origin and settling pattern of neurons of the lateral geniculate and lateral posterior nuclei. J Comp Neurol 284:581-601.

Baba K, Sakakibara S, Setsu T, Terashima T (2007) The superficial layers of the superior colliculus are cytoarchitectually and myeloarchitectually disorganized in the reelin-deficient mouse, reeler. Brain Res 1140:205-215.

Benhayon D, Magdaleno S, Curran T (2003) Binding of purified Reelin to ApoER2 and VLDLR mediates tyrosine phosphorylation of Disabled-1. Brain Res Mol Brain Res 112:33-45.

Bjartmar L, Huberman AD, Ullian EM, Rentería RC, Liu X, Xu W, Prezioso J, Susman MW, Stellwagen D, Stokes CC, Cho R, Worley P, Malenka RC, Ball S, Peachey NS, Copenhagen D, Chapman B, Nakamoto M, Barres BA, Perin MS (2006) Neuronal pentraxins mediate synaptic refinement in the developing visual system. J Neurosci 26:6269-6281.

Borrell V, Del Río JA, Alcántara S, Derer M, Martínez A, D’Arcangelo G, Nakajima K, Mikoshiba K, Derer P, Curran T, Soriano E (1999) Reelin regulates the development and synaptogenesis of the layer-specific entorhino-hippocampal connections. J Neurosci 19:1345-1358.

Borrell V, Pujadas L, Simó S, Durà D, Solé M, Cooper JA, Del Río JA, Soriano E (2007) Reelin and mDab1 regulate the development of hippocampal connections. Mol Cell Neurosci 36:158-173.

Botchkina GI, Morin LP (1995) Specialized neuronal and glial contributions to development of the hamster lateral geniculate complex and circadian visual system. J Neurosci 15:190-201.

Buhusi M, Demyanenko GP, Jannie KM, Dalal J, Darnell EP, Weiner JA, Maness PF (2009) ALCAM regulates mediolateral retinotopic mapping in the superior colliculus. J Neurosci 29:15630-15641.

Cohen-Cory S, Fraser SE (1994) BDNF in the development of the visual system of Xenopus. Neuron 12:747-761.

Cohen-Cory S, Fraser SE (1995) Effects of brain-derived neurotrophic factor on optic axon branching and remodelling in vivo. Nature 378:192-196.

D’Arcangelo G, Miao GG, Chen SC, Soares HD, Morgan JI, Curran T (1995) A protein related to extracellular matrix proteins deleted in the mouse mutant reeler. Nature 374:719-723. 
Datwani A, McConnell MJ, Kanold PO, Micheva KD, Busse B, Shamloo M, Smith SJ, Shatz CJ (2009) Classical MHCI molecules regulate retinogeniculate refinement and limit ocular dominance plasticity. Neuron 64:463-470.

Del Río JA, Heimrich B, Borrell V, Förster E, Drakew A, Alcántara S, Nakajima K, Miyata T, Ogawa M, Mikoshiba K, Derer P, Frotscher M, Soriano E (1997) A role for Cajal-Retzius cells and reelin in the development of hippocampal connections. Nature 385:70-74.

Demyanenko GP, Maness PF (2003) The L1 cell adhesion molecule is essential for topographic mapping of retinal axons. J Neurosci 23:530-538.

Ecker JL, Dumitrescu ON, Wong KY, Alam NM, Chen SK, LeGates T, Renna JM, Prusky GT, Berson DM, Hattar S (2010) Melanopsin-expressing retinal ganglion-cell photoreceptors: cellular diversity and role in pattern vision. Neuron 67:49-60.

Falconer DS (1951) 2 new mutants, trembler and reeler, with neurological actions in the house mouse (Mus musculus L). J Genet 50:192-201.

Feller MB (2009) Retinal waves are likely to instruct the formation of eyespecific retinogeniculate projections. Neural Dev 4:24.

Fox MA (2008) Novel roles for collagens in wiring the vertebrate nervous system. Curr Opin Cell Biol 20:508-513.

Fox MA, Sanes JR (2007) Synaptotagmin I and II are present in distinct subsets of central synapses. J Comp Neurol 503:280-296.

Frost DO, Edwards MA, Sachs GM, Caviness VS Jr (1986) Retinotectal projection in reeler mutant mice: relationships among axon trajectories, arborization patterns and cytoarchitecture. Brain Res 393:109-120.

Godement P, Salaün J, Imbert M (1984) Prenatal and postnatal development of retinogeniculate and retinocollicular projections in the mouse. J Comp Neurol 230:552-575.

Güler AD, Ecker JL, Lall GS, Haq S, Altimus CM, Liao HW, Barnard AR, Cahill H, Badea TC, Zhao H, Hankins MW, Berson DM, Lucas RJ, Yau KW, Hattar S (2008) Melanopsin cells are the principal conduits for rod-cone input to non-image-forming vision. Nature 453:102-105.

Hale PT, Sefton AJ (1978) A comparison of the visual and electrical response properties of cells in the dorsal and ventral lateral geniculate nuclei. Brain Res 153:591-595.

Hamel MG, Ajmo JM, Leonardo CC, Zuo F, Sandy JD, Gottschall PE (2008) Multimodal signaling by the ADAMTSs (a disintegrin and metalloproteinase with thrombospondin motifs) promotes neurite extension. Exp Neurol 210:428-440.

Harrington ME (1997) The ventral lateral geniculate nucleus and the intergeniculate leaflet: interrelated structures in the visual and circadian systems. Neurosci Biobehav Rev 21:705-727.

Hattar S, Liao HW, Takao M, Berson DM, Yau KW (2002) Melanopsincontaining retinal ganglion cells: architecture, projections, and intrinsic photosensitivity. Science 295:1065-1070.

Hattar S, Kumar M, Park A, Tong P, Tung J, Yau KW, Berson DM (2006) Central projections of melanopsin-expressing retinal ganglion cells in the mouse. J Comp Neurol 497:326-349.

Hindges R, McLaughlin T, Genoud N, Henkemeyer M, O’Leary DD (2002) EphB forward signaling controls directional branch extension and arborization required for dorsal-ventral retinotopic mapping. Neuron 35:475-487.

Howell BW, Hawkes R, Soriano P, Cooper JA (1997) Neuronal position in the developing brain is regulated by mouse disabled-1. Nature 389:733-737.

Huber AB, Kolodkin AL, Ginty DD, Cloutier JF (2003) Signaling at the growth cone: ligand-receptor complexes and the control of axon growth and guidance. Annu Rev Neurosci 26:509-563.

Huberman AD, Feller MB, Chapman B (2008a) Mechanisms underlying development of visual maps and receptive fields. Annu Rev Neurosci 31:479-509.

Huberman AD, Manu M, Koch SM, Susman MW, Lutz AB, Ullian EM, Baccus SA, Barres BA (2008b) Architecture and activity-mediated refinement of axonal projections from a mosaic of genetically identified retinal ganglion cells. Neuron 59:425-438.

Huberman AD, Wei W, Elstrott J, Stafford BK, Feller MB, Barres BA (2009) Genetic identification of an on-off direction-selective retinal ganglion cell subtype reveals a layer-specific subcortical map of posterior motion. Neuron 62:327-334.

Huberman AD, Clandinin TR, Baier H (2010) Molecular and cellular mechanisms of lamina-specific axon targeting. Cold Spring Harb Perspect Biol 2:a001743.
Huh GS, Boulanger LM, Du H, Riquelme PA, Brotz TM, Shatz CJ (2000) Functional requirement for class I MHC in CNS development and plasticity. Science 290:2155-2159.

Inoue A, Sanes JR (1997) Lamina-specific connectivity in the brain: regulation by $\mathrm{N}$-cadherin, neurotrophins, and glycoconjugates. Science 276:1428-1431.

Jacobs EC, Campagnoni C, Kampf K, Reyes SD, Kalra V, Handley V, Xie YY, Hong-Hu Y, Spreur V, Fisher RS, Campagnoni AT (2007) Visualization of corticofugal projections during early cortical development in a tauGFP-transgenic mouse. Eur J Neurosci 25:17-30.

Jaubert-Miazza L, Green E, Lo FS, Bui K, Mills J, Guido W (2005) Structural and functional composition of the developing retinogeniculate pathway in the mouse. Vis Neurosci 22:661-676.

Jones EG, Rubenstein JL (2004) Expression of regulatory genes during differentiation of thalamic nuclei in mouse and monkey. J Comp Neurol 477:55-80.

Kim IJ, Zhang Y, Yamagata M, Meister M, Sanes JR (2008) Molecular identification of a retinal cell type that responds to upward motion. Nature 452:478-482.

Kim IJ, Zhang Y, Meister M, Sanes JR (2010) Laminar restriction of retinal ganglion cell dendrites and axons: subtype-specific developmental patterns revealed with transgenic markers. J Neurosci 30:1452-1462.

Land PW, Kyonka E, Shamalla-Hannah L (2004) Vesicular glutamate transporters in the lateral geniculate nucleus: expression of VGLUT2 by retinal terminals. Brain Res 996:251-254.

Leamey CA, Merlin S, Lattouf P, Sawatari A, Zhou X, Demel N, Glendining KA, Oohashi T, Sur M, Fässler R (2007) Ten_m3 regulates eye-specific patterning in the mammalian visual pathway and is required for binocular vision. PLoS Biol 5:e241.

Lüth HJ, Winkelmann E, Celio MR (1993) Light- and electron microscopic localization of parvalbumin, calbindin D-28k and calretinin in the dorsal lateral geniculate nucleus of the rat. J Hirnforsch 34:47-56.

Masland RH (2001) Neuronal diversity in the retina. Curr Opin Neurobiol 11:431-436.

McFarlane S, McNeill L, Holt CE (1995) FGF signaling and target recognition in the developing Xenopus visual system. Neuron 15:1017-1028.

McFarlane S, Cornel E, Amaya E, Holt CE (1996) Inhibition of FGF receptor activity in retinal ganglion cell axons causes errors in target recognition. Neuron 17:245-254.

Moore RY, Speh JC (1993) GABA is the principal neurotransmitter of the circadian system. Neurosci Lett 150:112-116.

Morin LP, Blanchard J, Moore RY (1992) Intergeniculate leaflet and suprachiasmatic nucleus organization and connections in the golden hamster. Vis Neurosci 8:219-230.

Morin LP, Blanchard JH, Provencio I (2003) Retinal ganglion cell projections to the hamster suprachiasmatic nucleus, intergeniculate leaflet, and visual midbrain: bifurcation and melanopsin immunoreactivity. J Comp Neurol 465:401-416.

Muscat L, Huberman AD, Jordan CL, Morin LP (2003) Crossed and uncrossed retinal projections to the hamster circadian system. J Comp Neurol 466:513-524.

Nakagawa Y, O'Leary DD (2001) Combinatorial expression patterns of LIM-homeodomain and other regulatory genes parcellate developing thalamus. J Neurosci 21:2711-2725.

Niclou SP, Jia L, Raper JA (2000) Slit2 is a repellent for retinal ganglion cell axons. J Neurosci 20:4962-4974.

Pfeiffenberger C, Cutforth T, Woods G, Yamada J, Rentería RC, Copenhagen DR, Flanagan JG, Feldheim DA (2005) Ephrin-As and neural activity are required for eye-specific patterning during retinogeniculate mapping. Nat Neurosci 8:1022-1027.

Rebsam A, Petros TJ, Mason CA (2009) Switching retinogeniculate axon laterality leads to normal targeting but abnormal eye-specific segregation that is activity dependent. J Neurosci 29:14855-14863.

Rice DS, Curran T (2000) Disabled-1 is expressed in type AII amacrine cells in the mouse retina. J Comp Neurol 424:327-338.

Rice DS, Sheldon M, D’Arcangelo G, Nakajima K, Goldowitz D, Curran T (1998) Disabled-1 acts downstream of Reelin in a signaling pathway that controls laminar organization in the mammalian brain. Development 125:3719-3729.

Rice DS, Nusinowitz S, Azimi AM, Martínez A, Soriano E, Curran T (2001) The reelin pathway modulates the structure and function of retinal synaptic circuitry. Neuron 31:929-941. 
Sakuta H, Takahashi H, Shintani T, Etani K, Aoshima A, Noda M (2006) Role of bone morphogenic protein 2 in retinal patterning and retinotectal projection. J Neurosci 26:10868-10878.

Sanes JR, Yamagata M (2009) Many paths to synaptic specificity. Annu Rev Cell Dev Biol 25:161-195.

Sanes JR, Zipursky SL (2010) Design principles of insect and vertebrate visual systems. Neuron 66:15-36.

Schmitt AM, Shi J, Wolf AM, Lu CC, King LA, Zou Y (2006) Wnt-Ryk signalling mediates medial-lateral retinotectal topographic mapping. $\mathrm{Na}-$ ture 439:31-37.

Sheldon M, Rice DS, D’Arcangelo G, Yoneshima H, Nakajima K, Mikoshiba K, Howell BW, Cooper JA, Goldowitz D, Curran T (1997) Scrambler and yotari disrupt the disabled gene and produce a reeler-like phenotype in mice. Nature 389:730-733.

Steinbach K, Volkmer H, Schlosshauer B (2002) Semaphorin 3E/ collapsin-5 inhibits growing retinal axons. Exp Cell Res 279:52-61.

Stevens B, Allen NJ, Vazquez LE, Howell GR, Christopherson KS, Nouri N, Micheva KD, Mehalow AK, Huberman AD, Stafford B, Sher A, Litke AM, Lambris JD, Smith SJ, John SW, Barres BA (2007) The classical complement cascade mediates CNS synapse elimination. Cell 131:1164-1178.
Su J, Gorse K, Ramirez F, Fox MA (2010) Collagen XIX is expressed by interneurons and contributes to the formation of hippocampal synapses. J Comp Neurol 518:229-253.

Thankachan S, Rusak B (2005) Juxtacellular recording/labeling analysis of physiological and anatomical characteristics of rat intergeniculate leaflet neurons. J Neurosci 25:9195-9204.

Völgyi B, Chheda S, Bloomfield SA (2009) Tracer coupling patterns of the ganglion cell subtypes in the mouse retina. J Comp Neurol 512:664-687.

Webber CA, Hyakutake MT, McFarlane S (2003) Fibroblast growth factors redirect retinal axons in vitro and in vivo. Dev Biol 263:24-34.

Webber CA, Chen YY, Hehr CL, Johnston J, McFarlane S (2005) Multiple signaling pathways regulate FGF-2-induced retinal ganglion cell neurite extension and growth cone guidance. Mol Cell Neurosci 30:37-47.

Wu P, Li MS, Yu DM, Deng JB (2008) Reelin, a guidance signal for the regeneration of the entorhino-hippocampal path. Brain Res 1208:1-7.

Yamamoto T, Sakakibara S, Mikoshiba K, Terashima T (2003) Ectopic corticospinal tract and corticothalamic tract neurons in the cerebral cortex of yotari and reeler mice. J Comp Neurol 461:61-75. 